\title{
The Synthesizability of Molecules Proposed by Generative Models
}

\author{
Wenhao Gao ${ }^{\dagger, \uparrow}$ and Connor W. Coley ${ }^{*,+,+}$ \\ $\dagger$ Department of Chemical Engineering, MIT, Cambridge, MA \\ $\ddagger$ Broad Institute of Harvard and MIT, Cambridge, MA \\ \Department of Chemical and Biomolecular Engineering, Johns Hopkins University, \\ Baltimore, $M D$ \\ E-mail: ccoley@mit.edu
}

\begin{abstract}
The discovery of functional molecules is an expensive and time-consuming process, exemplified by the rising costs of small molecule therapeutic discovery. One class of techniques of growing interest for early-stage drug discovery is de novo molecular generation and optimization, catalyzed by the development of new deep learning approaches. ${ }^{1}$ These techniques can suggest novel molecular structures intended to maximize a multi-objective function, e.g., suitability as a therapeutic against a particular target, ${ }^{2]}$ without relying on brute-force exploration of a chemical space. ${ }^{3}$ However, the utility of these approaches is stymied by ignorance of synthesizability. To highlight the severity of this issue, we use a data-driven computer-aided synthesis planning pro$\operatorname{gram}^{4}$ to quantify how often molecules proposed by state-of-the-art generative models cannot be readily synthesized. Our analysis demonstrates that there are several tasks for which these models generate unrealistic molecular structures despite performing well on popular quantitative benchmarks. Synthetic complexity heuristics can successfully bias generation toward synthetically-tractable chemical space, although doing so
\end{abstract}


necessarily detracts from the primary objective. This analysis suggests that to improve the utility of these models in real discovery workflows, new algorithm development is warranted.

\section{Introduction}

Molecular design is one of the most fundamental challenges in chemical science and engineering. This task is to identify one or more molecules with a specific set of properties of interest, such as binding affinity and drug-likeness for drug design. High-throughput virtual screening (VS) is one widely used strategy to coarsely optimize a molecular structure using a discretized subspace of the whole chemical space. ${ }^{5}$ In VS, we evaluate enumerated candidate molecules in terms of their predicted properties of interest and ranked for follow-up experimental validation. However, because we rarely know a priori where the ideal molecule will be within the massive design space of chemical space, there is a trend toward using exceedingly large virtual libraries to increase the likelihood that we will find promising candidates. Modern virtual libraries may comprise hundreds of millions or billions of candidate molecules, ${ }^{3}$ often generated through combinatorial enumeration of commercially-available building block compounds. Even billions of compounds, however, represent a tiny fraction of theoretically-possible, pharmacologically-relevant small molecules, often cited as exceeding $10^{60}$ structures. ${ }^{[6}$ Brute-force virtual screening screening over a chemical space of this size is clearly computationally intractable.

Recent developments in computer aided drug design (CADD) techniques, especially in de novo molecular generation and optimization methods, raise the hope of removing this bottleneck. ${ }^{1]}$ Generative algorithms are a class of methods that propose molecular structures in a manner that can be tailored toward a specific objective. There is a long history of generative models in chemistry, many based on genetic algorithms ${ }^{7}$ and the iterative construction of molecules from molecular fragments. ${ }^{8}$ In the past decade, following on the advent of Variational Auto-Encoders (VAEs) ${ }^{9}$ and Generative Adversarial Networks (GANs), 10 there has 
been a flood of new deep learning (DL) methods for this task. ${ }^{11}$ Many of these methods learn a mapping from a continuous lower-dimensional real number space to a discrete chemical space. Jointly trained with a structure-property regression, one can obtain novel chemical structures conditioned on desired properties. More usefully, combining generative models with Bayesian optimization (BO), or directly using a heuristic optimization algorithm (e.g., a genetic algorithm (GA) or tree search (TS)), we can bias candidate generation toward the functionality we desire. Deep generative models are trained on a finite set of molecules to learn an underlying distribution of chemical space, where interpolation and extrapolation produce novel chemical structures. Enumerating every candidate molecule is thus unnecessary, and applying these models requires linear computational cost to generate multiple molecular structures once trained. Further, the generative algorithms can explore chemical space beyond the limited beginning pool and provide novel chemical structures with preferential intellectual property (IP) positions, whereas molecules in VS are often pre-existing. In recent years, generative models have been applied to various chemical discovery problems and have shown promise as a useful tool for the problem of molecular design. ${ }^{2}$

However, a practical problem that obstructs the usefulness of generative algorithms is that proposed molecular structures may be challenging or infeasible to synthesize. In any realistic discovery scenario, we will need to validate whether a proposed molecule has the property profile we expect; even if our computational models are infallible, we will need to manufacture the molecule in order to apply it (e.g., as a therapeutic, as a catalyst, as a component of a device). Libraries for virtual screening can be constructed from commerciallyavailable databases. They are often enumerated using well-characterized reaction templates to try to ensure that enumerated molecules are readily synthesizable. Lyu et al. report an $86 \%$ successfully synthesis rate among 51 top-ranking molecules from a library comprising 99 million structures, consistent with the claims of many chemical vendors. $\stackrel{3}{3}$

The situation is quite different in de novo molecular design, especially when using deep generative methods. We expect (and want) these models to explore molecular structures 
beyond the ones they have been trained on, so they may propose nonsensical structures that are unreasonable for pharmaceutical purposes. There have been few studies explicitly examining this problem, but some anecdotal evidence suggests that compounds are not easily synthesizable - many structures reported in papers indeed appear absurd. Bjerrum and Threlfall examined 21 molecules proposed by their recurrent neural network (RNN) model with Wiley's ChemPlanner and found a number of possible selectivity issues in the proposed syntheses, indicating synthetic difficulty. ${ }^{12}$ Sumita et al. filter generated molecules by requiring that they be previously reported with at least one synthetic route in SciFinder, which removes these models' ability to propose novel chemical structures. 13 Zhavoronkov et al. select only 6 molecules from 40 candidates structures based on synthetic accessibility, even after filtering an initial list of 30,000 structures generated by a deep learning model. ${ }^{2}$

Current procedures for quantifying synthesizability are based on (1) structure complexity and similarity or (2) synthetic pathways. The structure-based approach usually involves constructing a heuristic definition based on domain expertise or chemical substructure di-

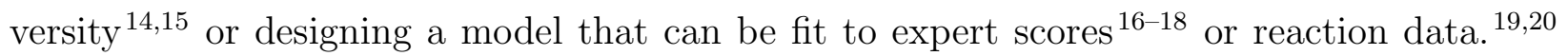
This kind of method is widely used due to its ease of implementation and low computational cost. However, two similar structures with a single functional group transposition can require substantially different synthetic routes (e.g., due to the selectivity of chemical reactions or availability of specific building blocks), which makes it challenging to fit a good proxy score (see Figure S1 and S2 for one example). The most convincing metric might be a direct scoring from a group of experts on synthetic, medicinal chemistry, which has been used as a ground truth to train models against. $\frac{16-19}{19}$ To have a group of experts that large enough to reach a non-biased and stable value is labor-intensive, hard to replicate, and not scalable. 21

The second, more nuanced approach to measuring synthesizability is to explicitly plan a synthetic pathway and assess its likelihood of experimental validity. Synthetic pathwaybased approaches can incorporate more thorough information about starting materials and chemical reactions, which enable them to overcome the shortcomings of the structure-based 
analysis. In this approach, a computer-aided synthesis planning (CASP) program 22 can be used to perform the retrosynthetic analysis. Using an explicit CASP tool is capable not only of capturing the high "non-linearity" of synthesizability with respect to chemical structure, but of recommending actionable synthetic pathways. We see this as a form of interpretability to verify why the molecule is believed to be synthesizable, with which building blocks, and in how many steps. Only a handful of studies have used a retrosynthetic planning tool to

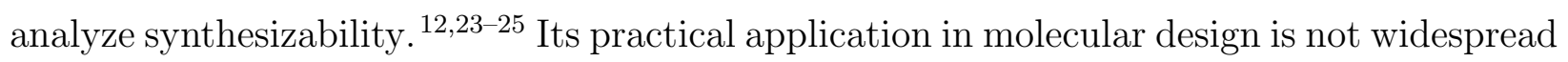
yet. Therefore, here, we analyze synthesizability of compounds proposed through generative algorithms using our open-source computer-aided retrosynthesis analysis tool, ASKCOS. ${ }^{4}$

We divide our analysis of the synthesizability of molecules generated by de novo generative algorithms into evaluations of distribution learning and goal-directed generation tasksunoptimized and optimized molecules, respectively. Distribution learning models are meant to interpolate within a chemical space comprised a training set of molecules and generate new molecules with similar properties. Goal-directed generation instead tries to generate new molecules that maximize a black-box scoring function. There are an increasing number of algorithms of these two categories proposed in recent years and a small number of studies that benchmark these algorithms in terms of their ability to generate novel, optimal molecules. $\underline{26 \mid 27}$

We categorize the approaches one might take to ensure that computationally designed molecules are able to be synthesized in Figure 1. These represent combinations of (i) a database of known or enumerated compounds, (ii) an evaluator, which estimates the properties we are trying to optimize, (iii) a generator function, which can propose new candidate molecules, (iv) a synthesizability oracle that determines whether it is straightforward to synthesize a given molecule, and/or (v) a heuristic synthesizability estimator that provides a computationally-inexpensive scalar measure of synthesizability. In this study, we focus on three major approaches to solving the synthesizability problem: post hoc filtering (Figure 17), imposing a priori differences in training sets (Figure 1 d), and heuristic biasing (Figure 1p). 


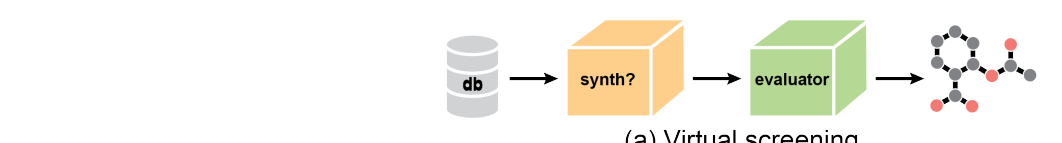

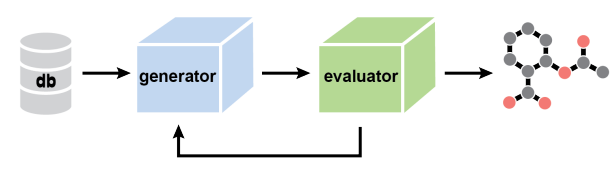

(b) Standard molecular generation

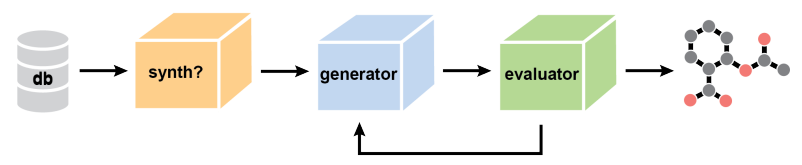

(d) Bias by training set

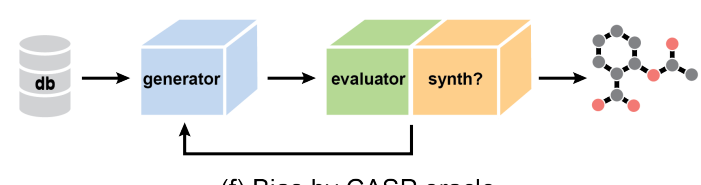

(f) Bias by CASP oracle

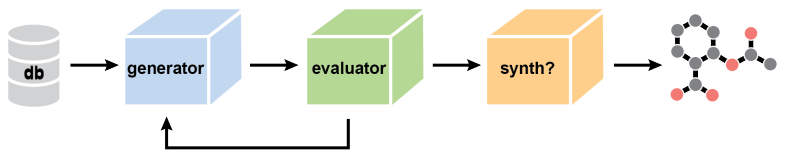

(c) Post hoc filter

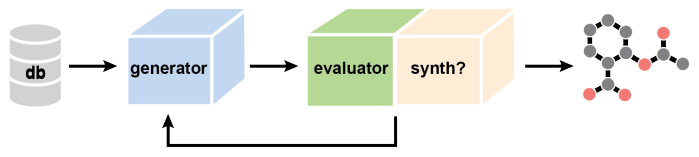

(e) Bias by heuristics

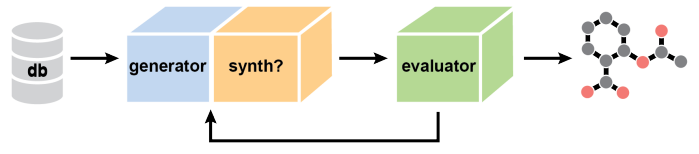

(g) Explicit constraints

Figure 1: Schematic representation of approaches to address the challenge of synthesizability in molecular optimization: (a) virtual screening can use a filtered database of candidates to ensure that they are all synthetically accessible; (b) standard molecular generation focuses on evaluation of properties without regard for synthesizability; (c) a post hoc filter narrows down proposed candidates as a separate step from generation; (d) biasing by training set aims to improve synthesizability by training generative models on synthetically-accessible compounds; (e) biasing by heuristics uses simple scalar proxies for synthesizability as part of the objective function; (f) biasing by a CASP oracle runs a full retrosynthetic expansion for proposed molecules to modify the reward function in a reinforcement learning setting; and (g) explicit constraints attempt to restrict chemical space to what is accessible using buyable building blocks and known synthetic transformations.

\section{Results}

\section{Synthesizability of common databases according to ASKCOS}

We first validate that the information returned by ASKCOS is usefully correlated with synthesizability by analyzing molecules from several standard compound libraries: MOSES, 26 ChEMBL, ${ }^{28}$ ZINC, ${ }^{29}$ Sheridan et al., 17 and GDB17 $7^{30}$ (see Methods for detailed descriptions of each data set and the settings used for retrosynthetic analysis, including the evaluation of commercial availability of building blocks). Figure 2 a shows the predicted number of synthetic steps required to produce a random set of 3000 molecules from each data set. The 
MOSES data set has the highest rate of perceived synthesizability at $89.8 \%$, consistent with its focus on small lead molecules and exclusion of compounds with "structural alerts". Its parent set, ZINC, has a lower synthesizability rate of $60.8 \%$. The ChEMBL data set has a higher rate of $68.3 \%$; although it contains larger and more complex structures than does ZINC, many have been synthesized previously; among those that cannot be synthesized are natural products that were extracted, not synthesized, and tested for their biological activity. ChEMBL also contains several directly purchasable compounds, second only to Sheridan et al.'s data set of 1730 compounds. Unsurprisingly, the exhaustively enumerated data set, GDB17, has the lowest rate of synthesizability at only 3.5\%. We also find that the predicted number of reaction steps is correlated with expert-provided scores (Figure S14). From these trends and the high success rate of the MOSES database, we conclude that ASKCOS's retrosynthetic analyses are largely consistent with our expectations of synthesizability and it is appropriate to use its predictions to benchmark the evaluation of molecular generation.

\section{Agreement between synthesizability heuristics and ASKCOS}

We next evaluate the agreement between several heuristic synthesizability scores (length of SMILES, SA_Score, $\frac{31}{21}$ and SCScore ${ }^{20}$ ) and the results of ASKCOS. Because retrosynthetic analysis can be time consuming (tens to hundreds of CPU-seconds), we would prefer to bias generation by heuristics rather than by a CASP oracle (cf. Figure 1). Figure 20-e show the trend of synthesizability of structures in different range of SA_Score, SCScore, and SMILES string length. None of them can distinguish the synthesizable and unsynthesizable compounds perfectly, but all exhibit a decreasing trend as the heuristic score increase. The trend is clearest for the SA_Score, followed by the SMILES length and then the SCScore. This ordering is quantified in Figure S3, which shows the area under the receiver operating characteristic as if these heuristics were being used for binary classification. The AUC values for the three methods in this order are $0.87,0.69$, and 0.61 . The slight shoulder around 5.5-6.0 is the contribution from the structurally complex but commercially available com- 
a
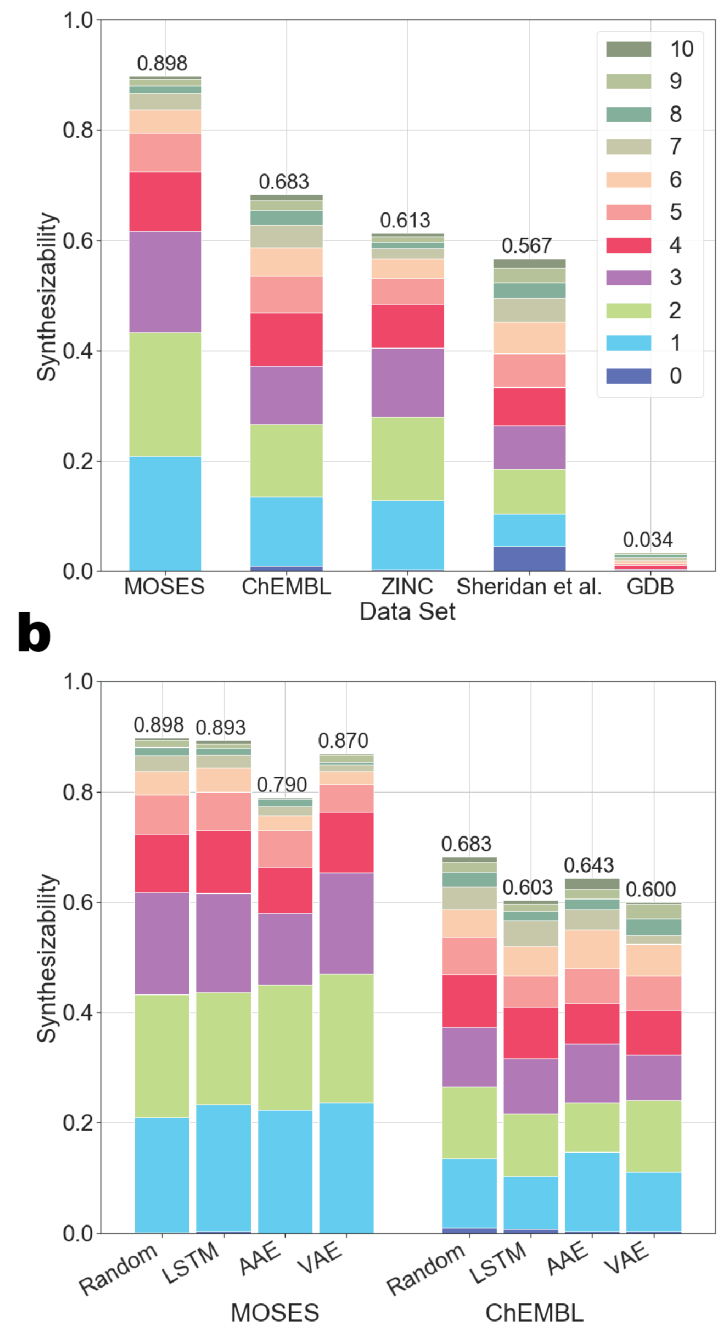

C

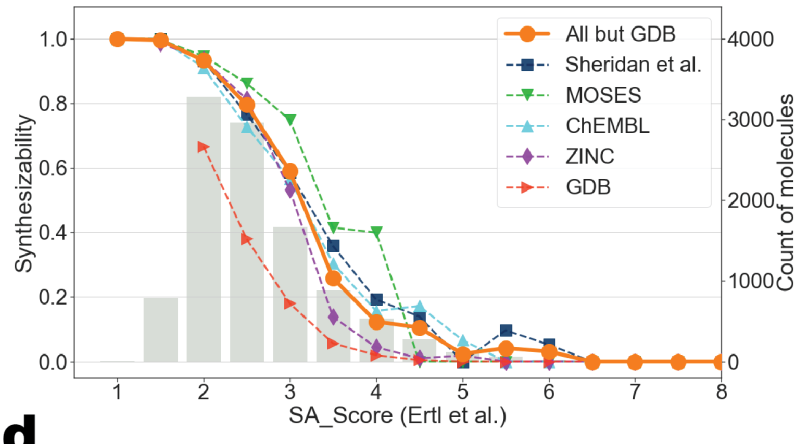

d
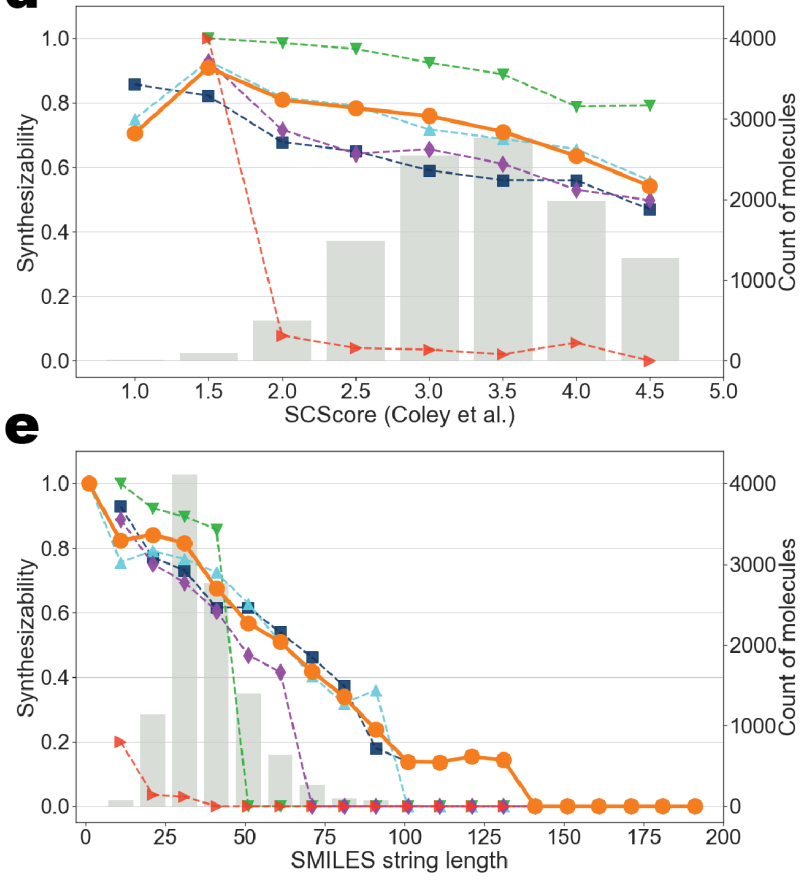

Figure 2: The synthesizability analysis of common data sets, distribution learning, and popular heuristics. (a) the number of synthetic steps required to produce random sampled structures from each data set; (b) the number of synthetic steps required to produce molecules generated by distribution learning algorithms, trained on either MOSES or ChEMBL; (c-e) the fraction of synthesizable compounds from each dataset binned by heuristic score and the number of molecules scored within each bin (excluding GDB).

pounds, highlighting the difference between synthetic complexity and structural complexity as discussed in ref. 20 , 


\section{Synthesizability of unoptimized generated molecules}

As alluded to above, distribution learning methods are capable of generating "unoptimized" molecules that share properties (in aggregate) with the database used for training. Here, we evaluate methods implemented in the $\operatorname{MOSES}^{26}$ benchmarking set, which cover diverse approaches to the molecular generation problem: a SMILES long short-term memory (LSTM) model, a variational auto-encoder (VAE), and an adversarial auto-encoder (AAE) (see Methods). There are more deep learning approaches for molecular generation and optimization than can be compared here, $\frac{11}{11}$ so we focus on these top-performing classes of approaches. In this task, we can use post hoc filtering or training set biasing by separately training distribution learning models on ChEMBL (less synthesizable) and MOSES (more synthesizable).

Figure $2 \mathrm{~b}$ shows the fraction of synthesizable molecules from 300 generated by each distribution learning method trained on the ChEMBL and MOSES. We observe that the fraction of synthesizable molecules are comparable to that of the training set, while no method improves synthesizability relative to its training set. The stark difference between results using MOSES and ChEMBL suggests that a priori biasing by training on a "more synthesizable" data set is a viable approach for distribution learning algorithms. There is no one method particularly superior than others. The high fraction of synthesizable results further suggests that post hoc filtering is not necessarily a bad approach (i.e., relatively few generated molecules would fail a check for synthesizability). Note these results pertain only to the synthesizability of generated results and do not consider previously evaluated metrics of novelty, uniqueness and diversity as do Polykovskiy et al.'s analyses. $\stackrel{26}{26}$

\section{Synthesizability of optimized generated molecules}

Our next analyses focus on goal-directed benchmarks, which reflect the actual use-case for generative models. Here, we re-evaluate the methods and objective functions evaluated by Brown et al.'s Guacamol ${ }^{27}$ in terms of their synthesizability. As detailed in the Methods, 


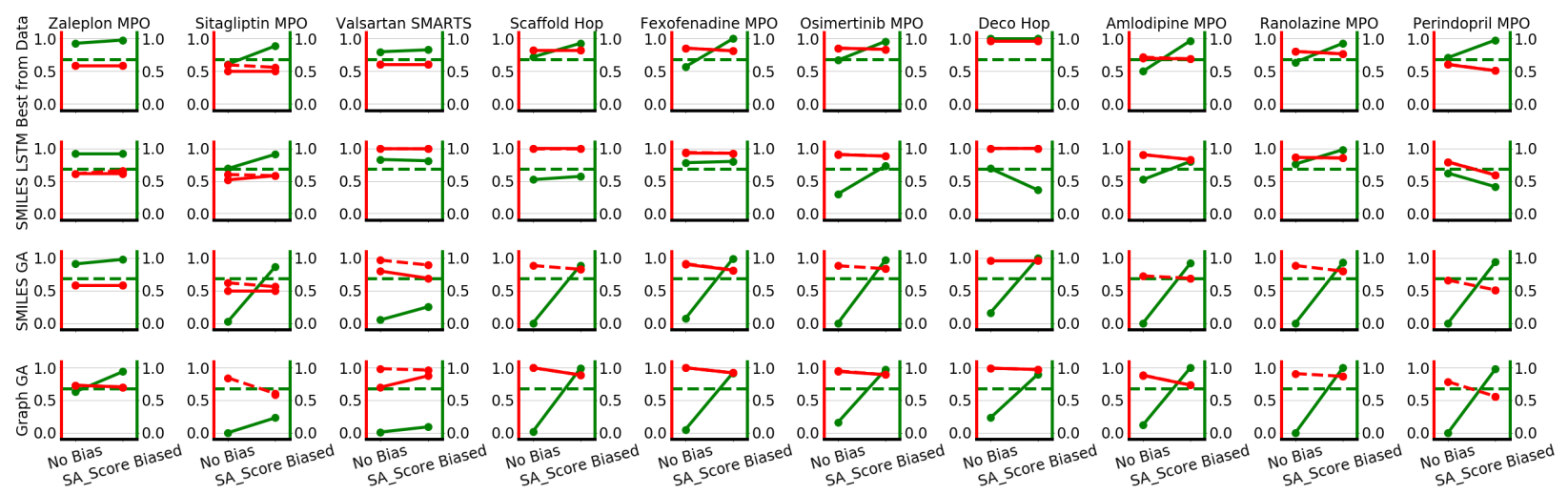

Figure 3: Dependence of goal-directed optimization performance on heuristic biasing by the SA_Score using ChEMBL as the training database. Each row represents result from one generative method; each column represents one objective function. In each plot, the green solid line represents the change of fraction of synthesizable compounds in the top-100 with the green dashed line as a reference for the synthesizability of the training set (ChEMBL). Red solid lines represent the change in the objective function value of the top synthesizable molecule, while the dashed red line represents the change in objective function value of the top molecule, regardless of its synthesizability. Plots without a solid red line indicates that no synthesizable structure was obtained in the top 100 molecules; dashed red lines may be occluded by solid red lines.

this includes three generative algorithms (SMILES LSTM, SMILES GA, and Graph GA) and 14 multi-property objective functions (MPOs) that convert a molecular structure to a scalar fitness score. As a baseline method, we include a virtual screening approach, "Best from Data", where all candidates from either ChEMBL or MOSES are evaluated to identify the top performers. In addition to post hoc filtering and training set biasing, we can also bias generation by modifying the objective function with a heuristic synthesizability score. We multiply the original objective functions (normalized between 0 and 1) with a quantitative synthesizability metric (SA_Score or SCScore, also normalized between 0 and 1). More details can be found in Methods section.

We evaluate the effects of heuristic biasing both in terms of the synthesizability of suggested molecules and in terms of the primary objective function value. Figure 3 shows how these metrics change when biasing with SA_Score, initially trained on ChEMBL (Figure S5 shows additional results using the SCScore/ChEMBL, SA_Score/MOSES, and SCScore/MOSES). Compared to the synthesizability of starting set (the green dashed lines), we 
a SMILES GA on Osimertinib MPO
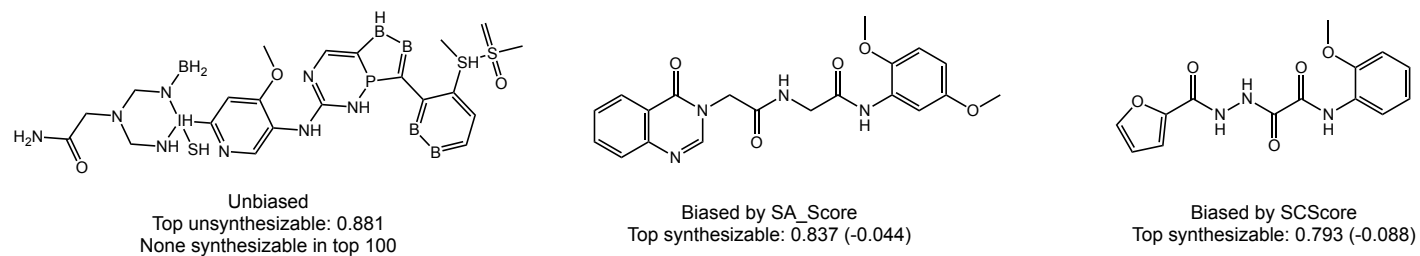

b Graph GA on Sitagliptin MPO
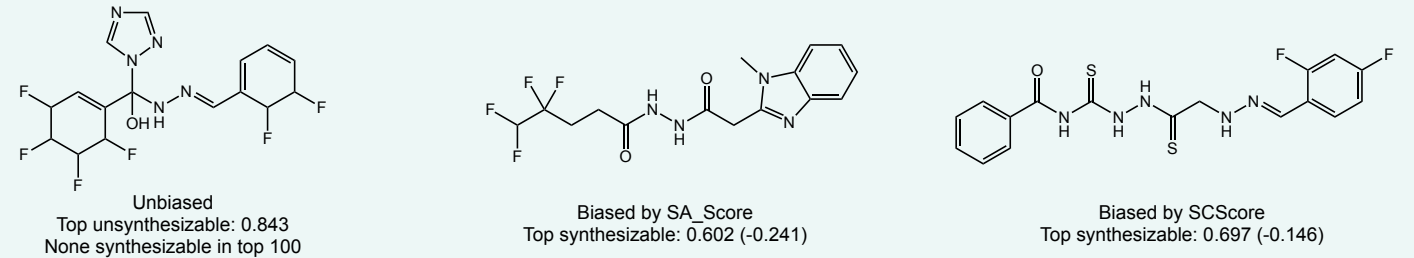

c Graph GA on Valsartan SMARTS
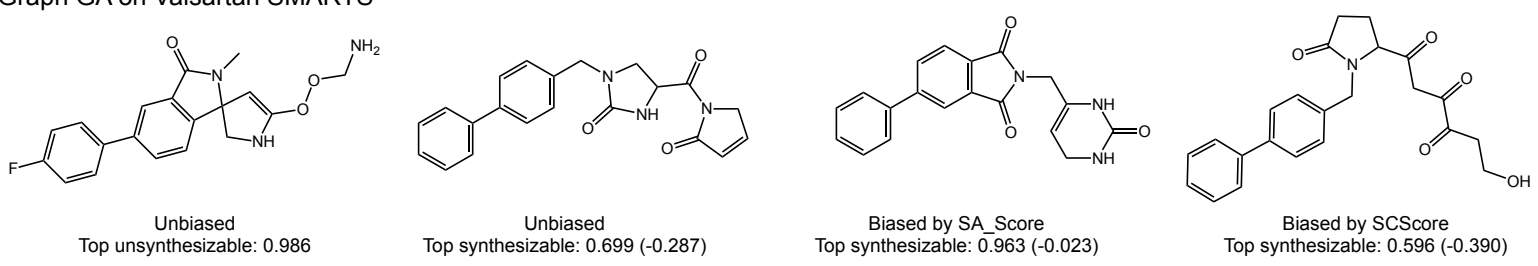

d Graph GA on Deco Hop
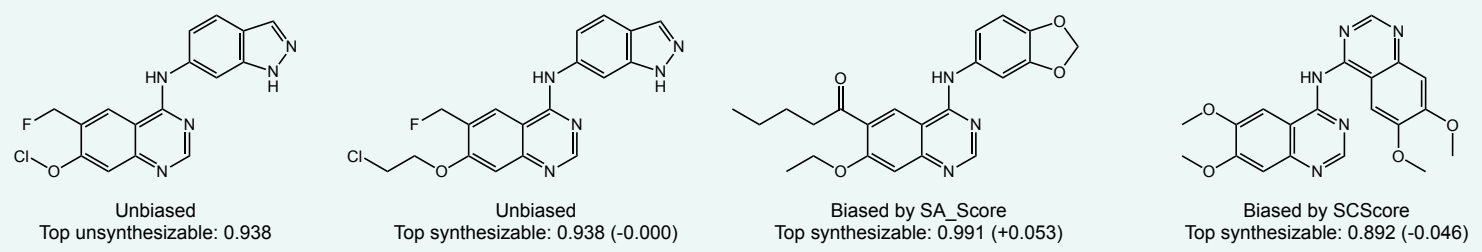

Figure 4: Examples of molecules from goal-directed optimization that were improved by heuristic biasing. Scores shown are the objective function values that have been normalized to the interval $[0,1]$. (a,b) cases where no synthesizable compounds were found in the top 100 suggestions without biasing, but at least one was found with either SA_Score or SCScore biasing. (c,d) cases where the top structure found without biasing was perceived as unsynthesizable and the use of heuristic biasing improved the objective function value of the top synthesizable structure.

can see the synthesizability varies between different methods and objectives. Indeed, the total fraction of synthesizable compounds in all methods for "hard" objectives without biasing is $30.2 \%$ with ChEMBL and $32.7 \%$ with MOSES (see Figure S6 for more details), excluding the direct sampling from data set. Compared to distribution learning, the goal-directed generation methods are less sensitive to the starting set of molecular compounds. For several tasks (Figure S6), very few or no compounds in the top 100 are synthesizable in the absence 
of heuristic biasing, particularly when using the genetic algorithms, illustrating the risk of relying on a post hoc filtering strategy. Examples in Figure 4 ab illustrate cases where no molecule in the top 100 is synthesizable and heuristic biasing is required to generate even a single feasible candidate. The compounds remaining after filtering for synthesizability, if any, may have low objective function values.

Most cases in Figure 3 show that the synthesizability of the top 100 compounds after biasing is quite high, often exceeding the rate for ChEMBL. Generally speaking, the SA_Score performs better than SCScore (Figure S5): the overall synthesizability for hard objectives was improved from $30.2 \%$ to $80.2 \%$ or $55.4 \%$ when biasing by SA_Score or SCScore, respectively, originally trained on ChEMBL (Table1). This successful result validates the approach shown in Figure11, but the increased synthesizability comes at the expense of the objective function value of the top candidate. For some tasks decreases by over $0.2-$ a significant difference for these benchmark tasks. However, we note that the value of an in silico objective function is completely inconsequential if the molecule cannot be made and experimentally tested.

Table 1: Fraction of synthesizable compounds in the top-100 candidates across all goaldirected optimization tasks and all methods, demonstrating successful heuristic biasing.

\begin{tabular}{llrrr}
\hline Training database & Task difficulty & Unbiased & Biased by SA_Score & Biased by SCScore \\
\hline \multirow{2}{*}{ ChEMBL } & trivial & $60.1 \%$ & $91.0 \%$ & $77.9 \%$ \\
& hard & $30.2 \%$ & $80.2 \%$ & $55.4 \%$ \\
\hline \multirow{2}{*}{ MOSES } & trivial & $63.5 \%$ & $92.2 \%$ & $78.8 \%$ \\
& hard & $32.7 \%$ & $77.2 \%$ & $58.0 \%$ \\
\hline
\end{tabular}

A fairer comparison can be made between the objective function values of the top synthesizable candidates, i.e., after post hoc filtering. Figure $4 \mathrm{~cd}$ shows two examples where the objective of the top- 1 candidate decreased, but the value of the top- 1 synthesizable candidate increased. That this is observed in some cases (also see Figure S13) suggests a practical workflow for molecular optimization: if only a few synthesizable candidates (1-10) are desired, first optimize without biasing and filter unsynthesizable suggestions; if the top synthesizable candidates are worse than the top unsynthesizable candidates, repeat the optimization while biasing with the SA_score. 


\section{Discussion of other approaches}

As described in Figure 1, there are more ways to improve synthesizability of de novo molecular generation algorithms. One promising approach is to bias the generation using a full CASP tool to evaluate synthesizability, instead of a proxy score (Figure 1f). The advantages are already described above; the disadvantage is the computational expense. While ASKCOS finds pathways in a few seconds for some molecules, we spend up to one minute evaluating each molecule to reduce the number of false negatives.

Benchmarking for molecular optimization, in addition to neglecting synthesizability, has largely neglected the number of objective function calls and computational expense. When using genetic algorithms for molecular optimization, we would first select high scoring synthesizable compounds as the initial set to propagate from a pool of up to millions of structures $\left(\sim 10^{6}\right)$ and then score, at each of hundreds of iterations $\left(\sim 10^{3}\right)$, hundreds of child compounds $\left(\sim 10^{3}\right)$. In total, we would require millions or at least hundreds of thousands of calls to the CASP oracle. Reinforcement-learning-based optimization methods that outperform Bayesian optimization when using VAEs require one oracle call per iteration, but require hundreds of thousands or millions of iterations (e.g., MolDQN reports the use of 200k function calls ${ }^{(32}$ ). One study by Korovina et al., who propose a method described in the next paragraph, highlight several existing methods that all require $\geq 5$ thousand evaluations for a single task compared to their 100. Based on the machine learning community's broader interest in improving the sample efficiency of reinforcement learning algorithms ${ }^{34}$ (thus fewer times calling the oracle) and CASP tools becoming faster, the use of an explicit retrosynthetic planner during optimization may become a computationally viable strategy.

The final approach (Figure $1 \mathrm{~g}$ ) is to embed synthesizability constraints in the generation algorithm itself, i.e., constrain the search space to molecules that can be produced from available building blocks. As early as 2003, Vinkers et al. describe the iterative optimization of molecular structure by selecting building blocks to react with a growing molecular structure. ${ }^{35}$ More recently, Bradshaw et al. ${ }^{36}$ propose a model called MoleculeChef that gen- 
erates a bag of reactants and uses a forward reaction prediction software to obtain the final products. Korovina et al.'s ChemBO similarly treats molecular generation as a random walk on a directed (synthetic) graph where each node is a molecule, and the parents of this node are the reagents that produce the child molecule when combined. $\frac{33}{3}$ These techniques are philosophically aligned with our use of retrosynthetic analysis to evaluate synthesizabilityboth try to use our collective knowledge of chemical reactivity to dictate what reactions are possible-but operate in the forward synthetic direction. This makes them subject to the same caveats that any CASP tool is subject to: their validity is entirely dependent on the accuracy of their forward reaction prediction engine, which can use either hand-coded rules or algorithmically-inferred rules. The greater the number of synthetic steps we allow, the lower the chances that each reaction will proceed as predicted. As this is essentially how virtual libraries are constructed, we would expect a similar rate of success (anecdotally, 85\% successful delivery of compounds from a library enumerated with a single synthetic step). Nevertheless, as the search space is directly constrained by these rules, they may enable a more efficient exploration of chemical space. We expect such algorithms to rapidly grow in popularity as the accuracy of reaction prediction tools improves. $\frac{37 / 38}{38}$

\section{Conclusion}

In this paper, we describe an analysis of the synthesizability of de novo generative algorithms. We first examined common chemical compound libraries and used ASKCOS to evaluate their synthesizability. We next evaluated molecules proposed by distribution learning and goal-directed generation methods, with and without biasing by heuristic synthesizability metrics. Distribution learning methods, provided they can learn the chemical distribution of the training set well, seem to generate molecules that are synthesizable with a similar frequency to their training set. Goal-directed generation methods have a significant risk of proposing unsynthesizable structures as their top suggestions, particularly using the SMILES 
GA or Graph GA methods, but occasionally there may be enough high-performing, synthesizable molecules in the top 100 that post hoc filtering (Figure 1ㄷ) is a viable strategy. In other cases, the proposed molecules are so absurd that one immediately recognizes why benchmarking these methods solely in terms of their objective function value is insufficient (e.g., Figure S10 and S11). Biasing generation by training set synthesizability (Figure 1 d) works for distribution learning, but does not have a noticeable effect on goal-directed optimization tasks. For some tasks, modifying the objective function with the SA_Score leads to candidates that outperform those obtained through post hoc filtering (Figure 4 $4 \mathrm{~d}$ and Figure S13). This heuristic biasing (Figure 17) almost always improves the synthesizability of generated candidates, but necessarily detracts from the main objective function.

We acknowledge that the identification of a synthetic pathway by ASKCOS is not a necessary or sufficient condition for synthesizability, nor would the generation of molecular candidates through forward synthesis prediction be a guarantee that those reactions would work experimentally. CASP tools for retrosynthesis and forward synthesis are imperfect. They do not capture our entire knowledge of chemical reactivity and may occasionally produce overly optimistic suggestions (e.g., with respect to selectivity). Further, the ability of CASP programs to find pathways is sensitive to the precise database of chemicals considered buyable and the settings one chooses for the retrosynthetic expansion. Even with an imperfect CASP tool like ASKCOS, however, we can obtain a meaningful analysis of synthesizability of generated molecules.

Generative models have a tremendous potential to accelerate molecular discovery. As we improve their ability to propose synthesizable molecules-whether by improving CASP tools for post hoc filtering, developing new heuristics for synthesizability, efficiently sampling a CASP oracle to bias generation with reinforcement learning, or designing new algorithms explicitly constrained by predictions of chemical reactivity-their utility and relevance to practical discovery projects will only increase. 


\section{Methods}

\section{ASKCOS}

ASKCOS is an open-source software framework that integrates efforts to generalize known chemistry to new substrates by learning to apply retrosynthetic transformations, to identify suitable reaction conditions, and to evaluate whether reactions are likely to

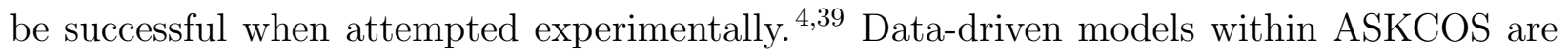
trained on millions of reactions from the U.S. Patent and Trademark Office (USPTO) and Reaxys databases. The core retrosynthetic capabilities rely on the recursive application of algorithmically-extracted reaction templates encoded as SMARTS patterns. Expansion is parallelized using an upper confidence bound tree search as detailed in the original publication. Importantly, ASKCOS has both programmatic and graphical interfaces to enable thousands of compounds to be processed without human intervention. The program makes extensive use of RDKit. $\underline{40}$

While the program offers flexible stopping criteria, we require starting materials to be commercially available according to a 2018 database of molecules from eMolecules or Sigma Aldrich with prices no greater than $\$ 100$ per gram; the full list is available in the ASKCOS codebase. This is a very strict price limit in the context of drug discovery, so it warrants two additional comments. First, one could consider most molecules to be "commercially available", in that some supplier or contract research organization will agree to produce them at some cost given sufficient lead time. Second, it is straightforward to modify the database of molecules considered commercially available depending on each user's price tolerance and available chemical inventory.

To determine whether a molecule is "synthesizable", we run a retrosynthetic expansion using ASKCOS with the following expansion settings: the maximum search depth-longest

linear sequence-is 9, the maximum branching ratio-number of unique precursors to consider at each disconnection-is 25 , the maximum wall time of expansion is 60 seconds, the maximum 
cumulative probability for target is 0.999 , the maximum number of templates to apply is 1000 , the maximum price for starting materials is $\$ 100 / \mathrm{g}$ as described above, the minimum plausibility of reactions-evaluated by a binary classifier as a "sanity check"-is 0.1. We terminate the search as soon as a pathway is found, rather than continuing to search for a more optimal (e.g., shorter, cheaper) pathway. All retrosynthetic analyses were carried out in an ASKCOS server on a debian virtual machine running on Google Cloud with 8 cores, 52 GB memory, and no other background tasks.

\section{Compound databases}

- $\operatorname{MOSES}^{\sqrt{26}}$ is an open database included in the MOSES benchmarking platform that evaluates distribution learning algorithms for drug discovery. The database of 1.94 million structures represents a subset of the 4.6 million in the ZINC Clean Leads collection with molar masses of $250-350 \mathrm{~g} / \mathrm{mol}$, fewer than 8 rotatable bonds, and a maximum XLogP of 3.5. Polykovskiy et al. filtered out molecules containing charged atoms, atoms besides $\mathrm{C}, \mathrm{N}, \mathrm{S}, \mathrm{O}, \mathrm{F}, \mathrm{Cl}, \mathrm{Br}$, and $\mathrm{H}$, cycles longer than 8 atoms, and molecules containing "structural alerts" from medicinal chemistry filters and PAINS filters.

- $\mathrm{ChEMBL}^{28}$ is a regularly-updated, open access database containing a large number of biologically-relevant compounds and associated assays (e.g., binding and ADMET). In our experiments, we use ChEMBL release 24, which contains 15.2 million activity measurements for 1.8 million compounds.

- ZINC 41 is an open database of commercially-available (not in-stock) compounds for virtual screening. ZINC contains over 230 million purchasable compounds in readyto-dock, 3D formats. We sampled molecules from ZINC-250k, which is a widely used subset of ZINC12 ${ }^{41}$ from Gómez-Bombarelli et al. ${ }^{42}$

- Sheridan et al. ${ }^{17}$ refers to a set of 1730 unique and parseable compounds taken from 
the 2575 unique molecules released by Merck in their paper exploring a crowdsourced definition of molecular complexity. These molecules were drawn from various public and Merck-internal sources as described in the original publication.

- GDB17 $7^{30}$ is an open database containing 166.4 billion enumerated molecules with up to 17 heavy atoms of $\mathrm{C}, \mathrm{N}, \mathrm{O}, \mathrm{S}$ and halogens. The enumeration started from mathematical graphs to form skeletons, aiming to cover size ranges containing many drugs and typical for lead compounds. We are sampling from its "Lead-like Set" of 800 thousand compounds with molar masses of 100-350 g/mol, CLogP of 1-3, and without 3- or 4-membered rings.

\section{Molecular generation algorithms}

- Random sampler is a baseline approach to molecular generation and optimization that randomly samples molecules (with replacement) from a "training set" of known compounds.

- Best from data represents the virtual screening approach to molecular optimization, where all molecules from a "training set" of known compounds are evaluated to identify the ones with the highest scores.

- $L S T M^{43}$ refers to a Long-Short Term Memory ${ }^{44}$ neural network that is widely used in natural language processing. The model is trained in an auto-regressive way to predict the next character of a simplified molecular-input line-entry (SMILES) string. It can be iteratively fine-tuned to optimize molecules toward a specific objective using a hill-climbing algorithm. We evaluated the implementation from ref. 27.

- $V A E^{42}$ refers to a variational autoencoder architecture that learns to construct a bidirectional mapping between SMILES represented chemical space and a finite-dimensional continuous latent space. The architecture is devised to learn a probabilistic generative 
model as well as its posterior, respectively known as decoder and encoder. The two parts are trained simultaneously by maximizing the evidence lower bound (ELBO) of the marginal likelihood, $\operatorname{ELBO}(\phi, \theta)=\mathbb{E}_{q_{\phi}(z \mid x)}\left[\log p_{\theta}(x \mid z)\right]-K L\left(q_{\phi}(z \mid x) \| p(z)\right)$, where $\phi$ and $\theta$ are differential parameters and KL is the Kullback-Leibler (KL) divergence. We evaluated the implementation from ref. 26 .

- $A A E^{45}$ is another approach to train a SMILES-based encoder-decoder architecture. Instead of KL regularization, AAE is trained with an adversarial learning regularization that matches the posterior distribution to a prior distribution. We evaluated the implementation from ref. 26.

- SMILES GA 4 is is a population-based grammar evolution algorithm. We evaluated Yoshikawa et al.'s model that adopted a "chromosome" with context-free grammar of SMILES string so that crossover and mutation happens at the level of SMILES tokens. Each "chromosome" can be decoded to a SMILES string and checked validity using . We evaluated the implementation from ref. 27.

- Graph $G A^{47}$ is another genetic algorithm that represents molecules as graphs, rather than relying on SMILES strings. The crossovers and mutations are performed by altering a molecular graph directly, i.e., exchanging substructures and hand-written substitution rules for mutation. We evaluated the implementation from ref. 27.

\section{Objective functions for optimization}

The suite of objective functions we use for goal-directed optimization were taken from Brown et al.'s benchmarking function sets. $\frac{27}{27}$ Evaluation is divided into "trivial" tasks and "hard" tasks following the language of the original work. The trivial tasks are named as such because almost all molecular optimization methods can perform exceedingly well on them (thus they are not suitable for the assessment of generative models), whereas the hard tasks 
show greater variation as a function of the method used. However, all of these objective functions are relatively simple heuristic functions of molecular structure.

The trivial objectives we use include quantitative estimate of drug-likeness (QED); $\frac{48}{2} \mathrm{a}$ central nervous system (CNS) MPO; hard objectives we use include Osimertinib MPO, Fexofenadine MPO, Ranolazine MPO, Perindopril MPO, Amlodipine MPO, Ranolazine MPO, Sitagliptin MPO, Zaleplon MPO, Valsartan SMARTS, Scaffold Hop and Decorator Hop. Some MPO tasks try to identify molecules dissimilar to the titular molecule but with similar properties; other MPO tasks try to identify molecules similar to the titular molecule but with "improved" druglikeness properties. We didn't include the benchmarks that measure the similarity to commercial drug molecules and isomer benchmarks in hard tasks because we think they are less meaningful for drug discovery purposes. We refer readers to the list of benchmarks in ref. 27 for a full description of these objectives.

\section{Biasing techniques for molecular generation}

- Post hoc filtering is the approach where a CASP tool is used to filter unsynthesizable molecules suggested by an unbiased generation. We evaluate this approach by calculating the fraction of molecules that would pass the ASKCOS filter and their objective function values.

- Training set biasing is the approach of starting with a molecule databases that has a higher fraction of synthesizable compounds as the training set for deep learning methods or the starting pool for genetic algorithms. In this paper, we use ChEMBL (68.3\% as tested) and MOSES (89.8\% as tested) as representative datasets with lower and higher synthesizabilities, respectively. This approach can be used in both unoptimized generation and optimized generation.

- Heuristic biasing is the approach of modifying the main objective function to penalize 
the generation of unsynthesizable compounds. We apply a synthesizability function multiplier, ranging from 0 to 1 , to a pre-normalized objective function (also ranging from 0 to 1). Specifically, we use a form of modified Gaussian and sigmoid function to rescale the heuristic score $x$ :

$$
\begin{gathered}
\text { Modifier }= \begin{cases}1 & x<\mu \\
e^{-\frac{(x-\mu)^{2}}{2 \sigma}} & x \geq \mu\end{cases} \\
\text { Modifier }=1-\frac{1}{1+e^{a(x-b)}}
\end{gathered}
$$

We performed 30 iterations of Tree Parzen Estimator (TPE) Bayesian Optimization to determine the hyper-parameters for each score. The hyper-parameters aimed to maximize the fraction of synthesizable suggestions times the average of the objective function for the top 10 molecules from graph genetic algorithm. We tested the biasing effect of SA_Score, SCScore, and length of SMILES string, but meaningful parameters could not be obtained for the SMILES string heuristic. The multipliers we use are shown in Figure S4. This approach can only be used in optimized generation.

- SA_Score ${ }^{31}$ is a popular heuristic score for quantifying synthesizability. It computes a score using a fragment-contribution approach, where rarer fragments (as judged by their abundance in the PubChem database) are taken as an indication of lower synthesizability.

- SCScore ${ }^{20}$ is a learned synthetic complexity score computed by as neural network model trained on reaction data from the Reaxys database. It was designed with synthesis planning in mind to operate on molecules resembling not just drug-like products, but intermediates and simpler building blocks as well.

- SMILES length is a very simple heuristic that associates molecules with longer SMILES strings as an indication of synthetic difficulty. The length of a SMILES 
string correlates closely with the number of heavy atoms in a molecule (i.e., larger molecules are harder to synthesize), but is further increased by the presence of formal charges, ring closures, and defined stereochemistry.

\section{Acknowledgement}

This work was supported by the Machine Learning for Pharmaceutical Discovery and Synthesis consortium. We thank Mike Fortunato and Thomas Struble for assisting with programmatic interfacing of ASKCOS. We also thank Lagnajit Pattanaik and Klavs Jensen for commenting on the manuscript.

\section{Supporting Information Available}

The following files are available free of charge.

All code and data can be found at https://github.com/wenhao-gao/askcos_synthesizability. Additional results can be found in the supporting information.

\section{References}

(1) Sanchez-Lengeling, B.; Aspuru-Guzik, A. Inverse molecular design using machine learning:Generative models for matter engineering. Science 2018, 361, 360-365.

(2) Zhavoronkov, A.; Ivanenkov, Y. A.; Aliper, A.; Veselov, M. S.; Aladinskiy, V. A.; Aladinskaya, A. V.; Terentiev, V. A.; Polykovskiy, D. A.; Kuznetsov, M. D.; Asadulaev, A., et al. Deep learning enables rapid identification of potent DDR1 kinase inhibitors. Nat. Biotechnol. 2019, 37, 1038-1040.

(3) Lyu, J.; Wang, S.; Balius, T. E.; Singh, I.; Levit, A.; Moroz, Y. S.; O'Meara, M. J.; Che, T.; Algaa, E.; Tolmachova, K.; Tolmachev, A. A.; Shoichet, B. K.; Roth, B. L.; 
Irwin, J. J. Ultra-large library docking for discovering new chemotypes. Nature 2019, 1.

(4) Coley, C. W.; Thomas, D. A.; Lummiss, J. A.; Jaworski, J. N.; Breen, C. P.; Schultz, V.; Hart, T.; Fishman, J. S.; Rogers, L.; Gao, H., et al. A robotic platform for flow synthesis of organic compounds informed by AI planning. Science 2019, 365, eaax1566.

(5) Walters, W. P.; Wang, R. New Trends in Virtual Screening. J. Chem. Inf. Model 2019, 59, 3603-3604.

(6) Virshup, A. M.; Contreras-García, J.; Wipf, P.; Yang, W.; Beratan, D. N. Stochastic voyages into uncharted chemical space produce a representative library of all possible drug-like compounds. J. Am. Chem. Soc 2013, 135, 7296-7303.

(7) Lameijer, E.-W.; Bäck, T.; Kok, J. N.; Ijzerman, A. P. Evolutionary algorithms in drug design. Natural Computing 2005, 4, 177-243.

(8) Lewis, D. W.; Willock, D. J.; Catlow, C. R. A.; Thomas, J. M.; Hutchings, G. J. De novo design of structure-directing agents for the synthesis of microporous solids. Nature 1996, 382, 604.

(9) Kingma, D. P.; Welling, M. Auto-encoding variational bayes. arXiv preprint $\operatorname{arXiv:1312.61142013,}$

(10) Goodfellow, I.; Pouget-Abadie, J.; Mirza, M.; Xu, B.; Warde-Farley, D.; Ozair, S.; Courville, A.; Bengio, Y. Generative adversarial nets. Advances in Neural Information Processing Systems. 2014; pp 2672-2680.

(11) Elton, D. C.; Boukouvalas, Z.; Fuge, M. D.; Chung, P. W. Deep learning for molecular generation and optimization - a review of the state of the art. arXiv:1903.04388 [physics, stat] 2019, arXiv: 1903.04388. 
(12) Bjerrum, E. J.; Threlfall, R. Molecular Generation with Recurrent Neural Networks (RNNs). arXiv:1705.04612 [cs, q-bio] 2017, arXiv: 1705.04612.

(13) Sumita, M.; Yang, X.; Ishihara, S.; Tamura, R.; Tsuda, K. Hunting for Organic Molecules with Artificial Intelligence: Molecules Optimized for Desired Excitation Energies. ACS Cent. Sci. 2018, 4, 1126-1133.

(14) Bertz, S. H. The First General Index of Molecular Complexity. J. Am. Chem. Soc. 1981, 103, 3599-3601.

(15) Ertl, P.; Schuffenhauer, A. Estimation of Synthetic Accessibility Score of Drug-Like Molecules Based on Molecular Complexity and Fragment Contributions. J. Cheminform. 2009, 1, 8 .

(16) Takaoka, Y.; Endo, Y.; Yamanobe, S.; Kakinuma, H.; Okubo, T.; Shimazaki, Y.; Ota, T.; Sumiya, S.; Yoshikawa, K. Development of a method for evaluating druglikeness and ease of synthesis using a data set in which compounds are assigned scores based on chemists' intuition. J. Chem. Inf. Model 2003, 43, 1269-1275.

(17) Sheridan, R. P.; Zorn, N.; Sherer, E. C.; Campeau, L.-C.; Chang, C.; Cumming, J.; Maddess, M. L.; Nantermet, P. G.; Sinz, C. J.; OŚhea, P. D. Modeling a Crowdsourced Definition of Molecular Complexity. J. Chem. Inf. Model. 2014, 54, 1604-1616.

(18) Baba, Y.; Isomura, T.; Kashima, H. Wisdom of crowds for synthetic accessibility evaluation. J. Mol. Graph. Model. 2018, 80, 217-223.

(19) Li, J.; Eastgate, M. D. Current Complexity: a Tool for Assessing the Complexity of Organic Molecules. Org. Biomol. Chem. 2015, 13, 7164-7176.

(20) Coley, C. W.; Rogers, L.; Green, W. H.; Jensen, K. F. SCScore: Synthetic complexity learned from a reaction corpus. J. Chem. Inf. Model 2018, 58, 252-261. 
(21) Lajiness, M. S.; Maggiora, G. M.; Shanmugasundaram, V. Assessment of the consistency of medicinal chemists in reviewing sets of compounds. J. Med. Chem. 2004, 47, 4891-4896.

(22) Feng, F.; Lai, L.; Pei, J. Computational chemical synthesis analysis and pathway design. Front. Chem. 2018, 6, 199.

(23) Podolyan, Y.; Walters, M. A.; Karypis, G. Assessing synthetic accessibility of chemical compounds using machine learning methods. J. Chem. Inf. Model 2010, 50, 979-991.

(24) Huang, Q.; Li, L.-L.; Yang, S.-Y. RASA: a rapid retrosynthesis-based scoring method for the assessment of synthetic accessibility of drug-like molecules. J. Chem. Inf. Model 2011, 51, 2768-2777.

(25) Bonnet, P. Is chemical synthetic accessibility computationally predictable for drug and lead-like molecules? A comparative assessment between medicinal and computational chemists. Eur. J. Med. Chem. 2012, 54, 679-689.

(26) Polykovskiy, D.; Zhebrak, A.; Sanchez-Lengeling, B.; Golovanov, S.; Tatanov, O.; Belyaev, S.; Kurbanov, R.; Artamonov, A.; Aladinskiy, V.; Veselov, M.; Kadurin, A.; Nikolenko, S.; Aspuru-Guzik, A.; Zhavoronkov, A. Molecular Sets (MOSES): A Benchmarking Platform for Molecular Generation Models. arXiv:1811.12823 [cs, stat] 2018, arXiv: 1811.12823.

(27) Brown, N.; Fiscato, M.; Segler, M. H. S.; Vaucher, A. C. GuacaMol: Benchmarking Models for De Novo Molecular Design. arXiv:1811.09621 [physics, q-bio] 2018, arXiv: 1811.09621.

(28) Gaulton, A.; Bellis, L. J.; Bento, A. P.; Chambers, J.; Davies, M.; Hersey, A.; Light, Y.; McGlinchey, S.; Michalovich, D.; Al-Lazikani, B.; Overington, J. P. ChEMBL: a largescale bioactivity database for drug discovery. Nucleic Acids Res. 2012, 40, D1100D1107. 
(29) Sterling, T.; Irwin, J. J. ZINC 15 - Ligand Discovery for Everyone. J. Chem. Inf. Model 2015, 55, 2324-2337.

(30) Ruddigkeit, L.; van Deursen, R.; Blum, L. C.; Reymond, J.-L. Enumeration of 166 Billion Organic Small Molecules in the Chemical Universe Database GDB-17. J. Chem. Inf. Model 2012, 52, 2864-2875.

(31) Ertl, P.; Schuffenhauer, A. Estimation of synthetic accessibility score of drug-like molecules based on molecular complexity and fragment contributions. J. Cheminform. $2009,1,8$.

(32) Zhou, Z.; Kearnes, S.; Li, L.; Zare, R. N.; Riley, P. Optimization of molecules via deep reinforcement learning. Scientific reports 2019, 9, 1-10.

(33) Korovina, K.; Xu, S.; Kandasamy, K.; Neiswanger, W.; Poczos, B.; Schneider, J.; Xing, E. P. ChemBO: Bayesian Optimization of Small Organic Molecules with Synthesizable Recommendations. arXiv preprint arXiv:1908.01425 2019,

(34) Ortega, P. A.; Wang, J. X.; Rowland, M.; Genewein, T.; Kurth-Nelson, Z.; Pascanu, R.; Heess, N.; Veness, J.; Pritzel, A.; Sprechmann, P., et al. Meta-learning of sequential strategies. arXiv preprint arXiv:1905.03030 2019,

(35) Vinkers, H. M.; de Jonge, M. R.; Daeyaert, F. F. D.; Heeres, J.; Koymans, L. M. H.; van Lenthe, J. H.; Lewi, P. J.; Timmerman, H.; Van Aken, K.; Janssen, P. A. J. SYNOPSIS: SYNthesize and OPtimize System in Silico. J. Med. Chem. 2003, 46, 2765-2773.

(36) Bradshaw, J.; Paige, B.; Kusner, M. J.; Segler, M. H.; Hernández-Lobato, J. M. A Model to Search for Synthesizable Molecules. arXiv preprint arXiv:1906.05221 2019,

(37) Coley, C. W.; Jin, W.; Rogers, L.; Jamison, T. F.; Jaakkola, T. S.; Green, W. H.; Barzilay, R.; Jensen, K. F. A graph-convolutional neural network model for the prediction of chemical reactivity. Chem. Sci. 2019, 10, 370-377. 
(38) Schwaller, P.; Laino, T.; Gaudin, T.; Bolgar, P.; Bekas, C.; Lee, A. A. Molecular Transformer for Chemical Reaction Prediction and Uncertainty Estimation. 2018,

(39) ASKCOS Software Repository. https://github.com/connorcoley/ASKCOS.

(40) Landrum, G. RDKit: Open-Source Cheminformatics Software. 2016,

(41) Irwin, J. J.; Sterling, T.; Mysinger, M. M.; Bolstad, E. S.; Coleman, R. G. ZINC: a free tool to discover chemistry for biology. J. Chem. Inf. Model 2012, 52, 1757-1768.

(42) Gómez-Bombarelli, R.; Wei, J. N.; Duvenaud, D.; Hernández-Lobato, J. M.; SánchezLengeling, B.; Sheberla, D.; Aguilera-Iparraguirre, J.; Hirzel, T. D.; Adams, R. P.; Aspuru-Guzik, A. Automatic chemical design using a data-driven continuous representation of molecules. ACS Cent. Sci. 2018, 4, 268-276.

(43) Segler, M. H.; Kogej, T.; Tyrchan, C.; Waller, M. P. Generating focused molecule libraries for drug discovery with recurrent neural networks. ACS Cent. Sci. 2017, 4, $120-131$.

(44) Hochreiter, S.; Schmidhuber, J. Long short-term memory. Neural Computation 1997, 9, 1735-1780.

(45) Polykovskiy, D.; Zhebrak, A.; Vetrov, D.; Ivanenkov, Y.; Aladinskiy, V.; Mamoshina, P.; Bozdaganyan, M.; Aliper, A.; Zhavoronkov, A.; Kadurin, A. Entangled conditional adversarial autoencoder for de novo drug discovery. Molecular pharmaceutics 2018, $15,4398-4405$.

(46) Yoshikawa, N.; Terayama, K.; Sumita, M.; Homma, T.; Oono, K.; Tsuda, K. Population-based de novo molecule generation, using grammatical evolution. Chem. Lett. 2018, 47, 1431-1434.

(47) Jensen, J. H. A graph-based genetic algorithm and generative model/Monte Carlo tree search for the exploration of chemical space. Chem. Sci. 2019, 10, 3567-3572. 
(48) Bickerton, G. R.; Paolini, G. V.; Besnard, J.; Muresan, S.; Hopkins, A. L. Quantifying the chemical beauty of drugs. Nat. Chem. 2012, 4, 90 .

(49) Wager, T. T.; Hou, X.; Verhoest, P. R.; Villalobos, A. Central nervous system multiparameter optimization desirability: application in drug discovery. ACS Chem. Neurosci. 2016, 7, 767-775. 


\section{Supporting Information}

\section{The Synthesizability of Molecules Proposed by Generative Models}

Wenhao Gao and Connor W. Coley*

Department of Chemical Engineering, MIT, Cambridge, MA 02139

Broad Institute of Harvard and MIT, Cambridge, MA 02139

Department of Chemical and Biomolecular Engineering, Johns Hopkins University, Baltimore, MD 21218

E-mail: ccoley@mit.edu

Code

All code can be found at https://github.com/wenhao-gao/askcos_synthesizability.

\section{Additional Results}

Suitability of heuristic functions for estimating synthesizability 


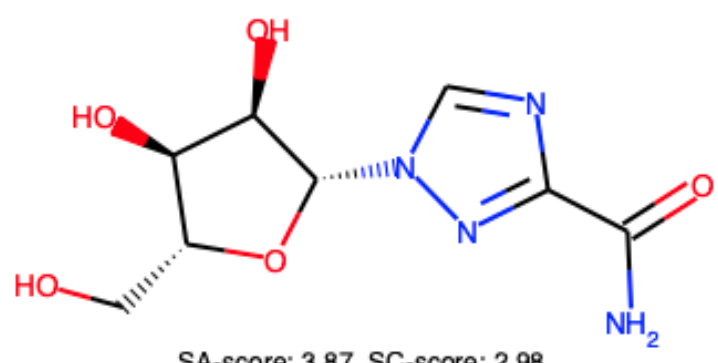

SA-score: 3.87, SC-score: 2.98

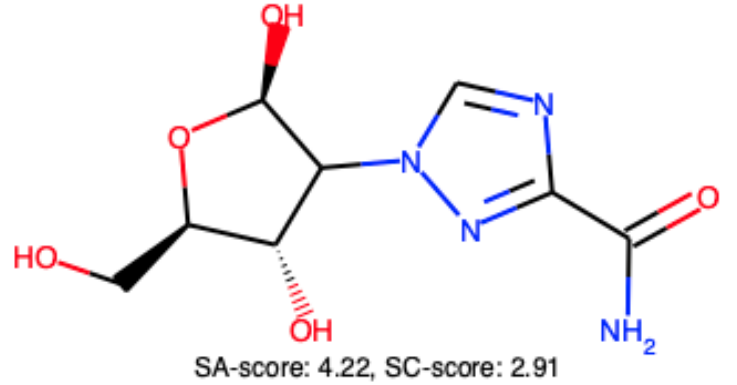

SA-score: 4.22, SC-score: 2.91

Figure S1: Illustration of the difficulty of applying heuristics to estimate synthesizability. Ribavirin (left) and its analogue (right) are structurally very similar, but their syntheses would be substantially different due to the inherent reactivity of ribose to favor substitution at the position leading to ribavirin. The SA_Score and SCScore do not reflect that the righthand compound is much harder to access. 


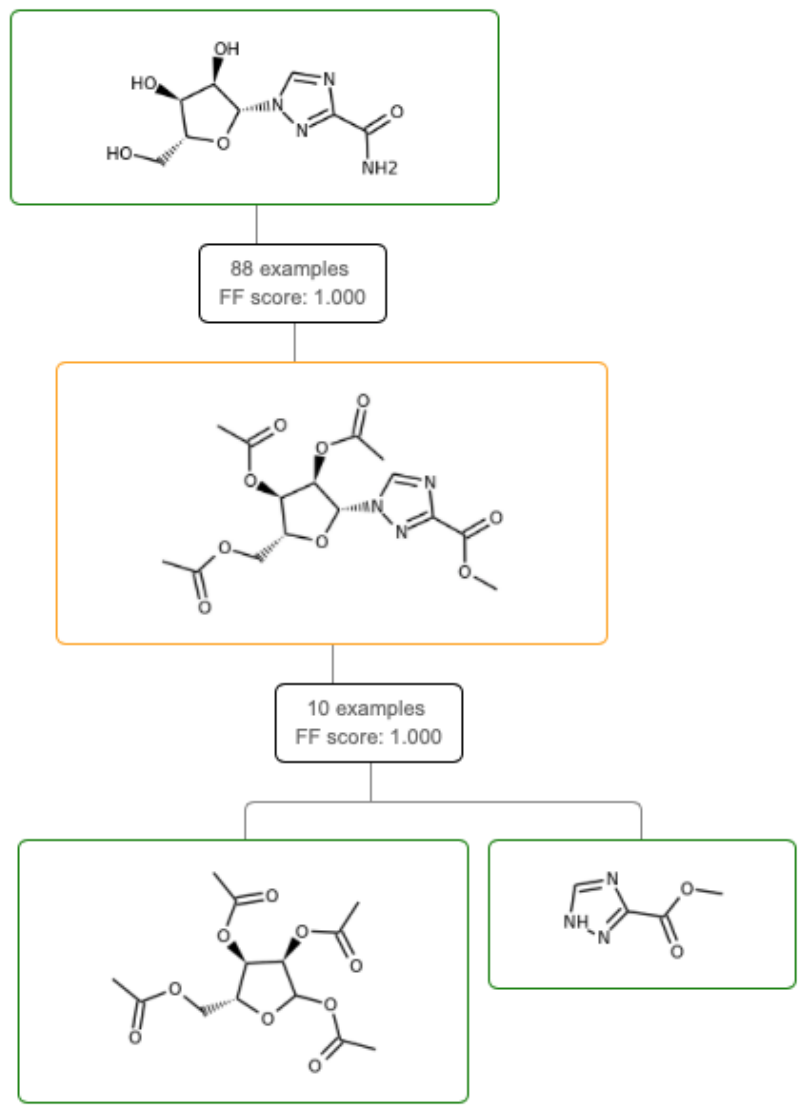

Figure S2: The synthetic pathway found by ASKCOS for ribavirin (left of Figure S1). By explicitly planning synthetic routes, ASKCOS easily distinguishes between the two compounds as it cannot identify a synthetic pathway for the righthand compound. 


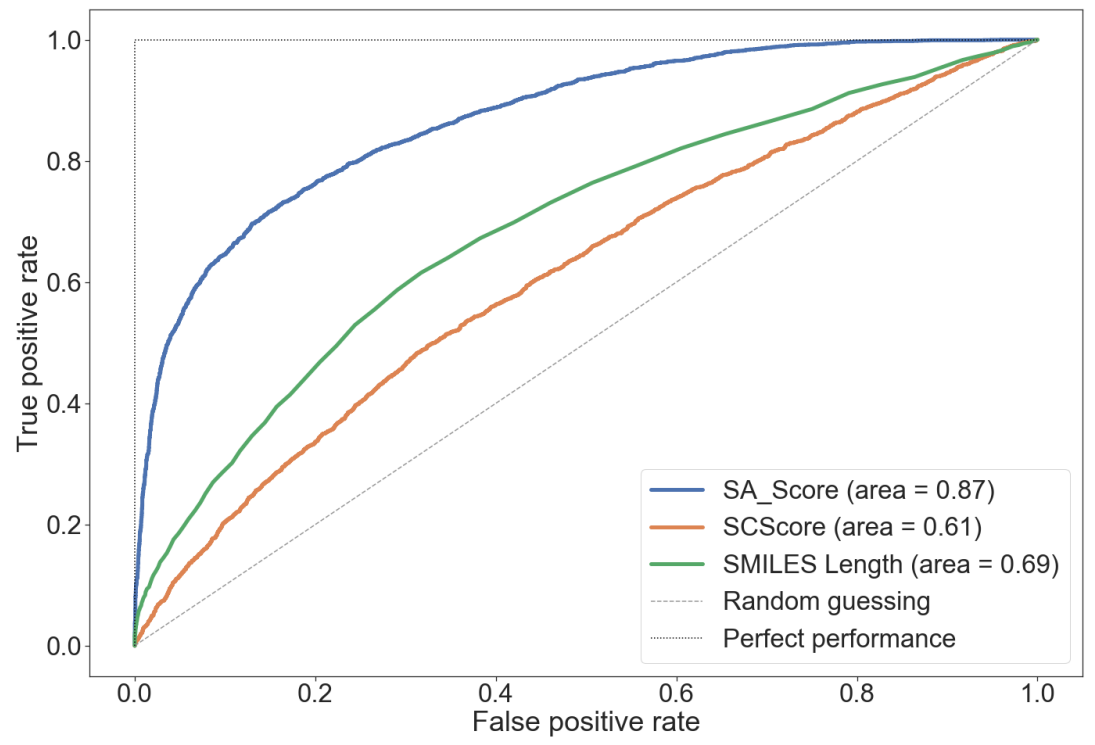

Figure S3: The receiver operating characteristic (ROC) curve obtained when using heuristic estimates of synthetic complexity for binary classification of molecules in the "All but GDB" compound set as synthesizable or unsynthesizable as perceived by ASKCOS. The area under the curve (AUC) quantifies the visual trends observed in Figure $2 \mathrm{c}$-e. On this compound set, the SA_Score outperforms the SMILES heuristic, which outperforms the SCScore. All three are better than randomly guessing. 


\section{Full results of heuristics biasing in goal-directed generation}
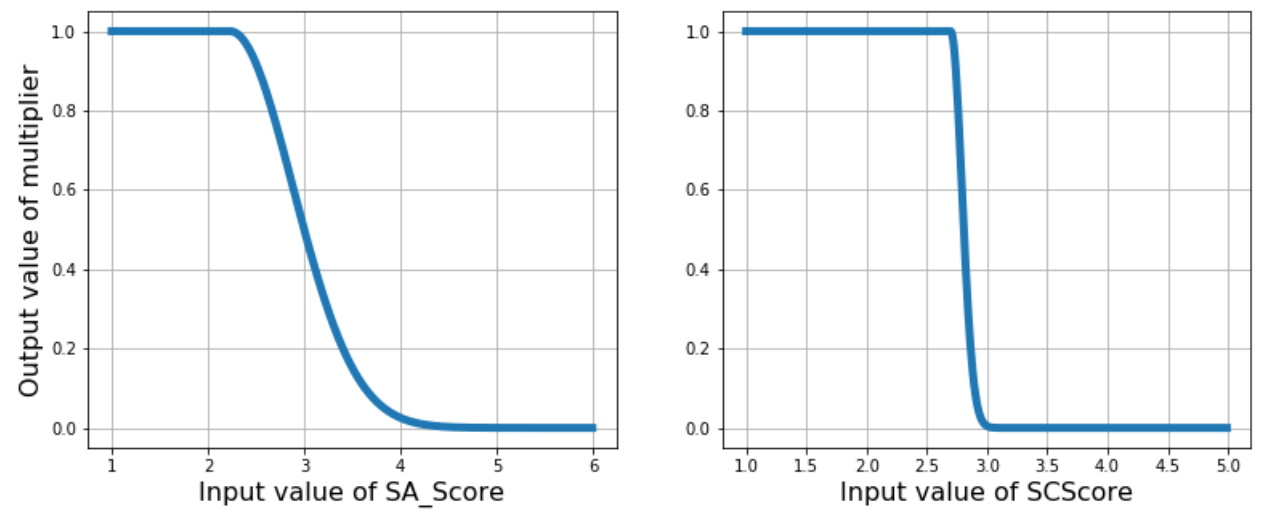

Figure S4: The scaled synthesizability multipliers used for heuristic biasing after optimizing shape parameters $(\mu$ and $\sigma$ ) (see Methods). 
a Z Zaleplon MPO Sitagliptin MPO Valsartan SMARTS Scaffold Hop Fexofenadine MPO Osimertinib MPO Deco Hop Amlodipine MPO Ranolazine MPO Perindopril MPO

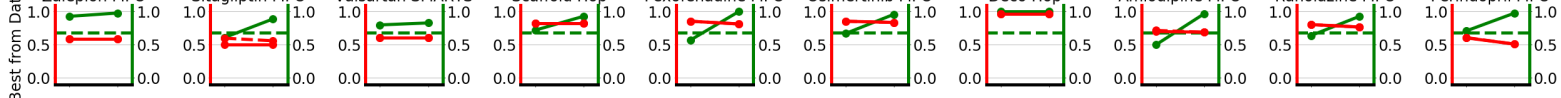

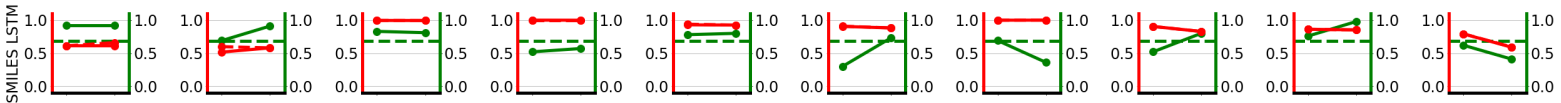

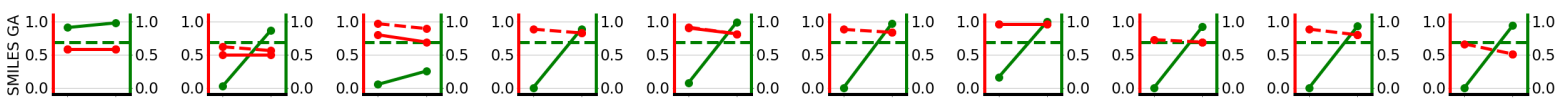

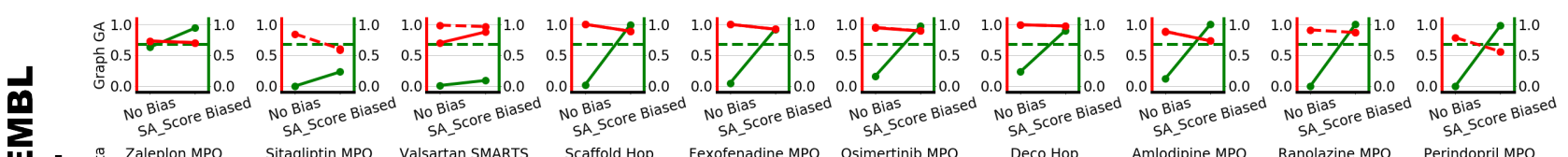
UD b

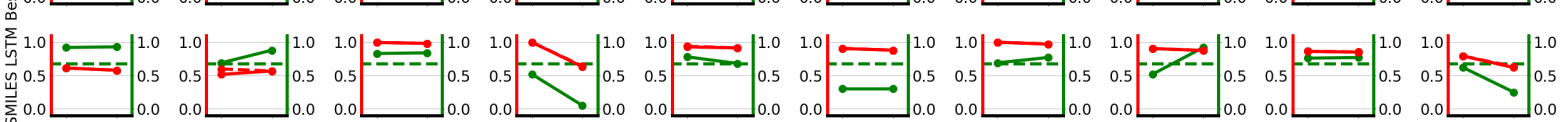

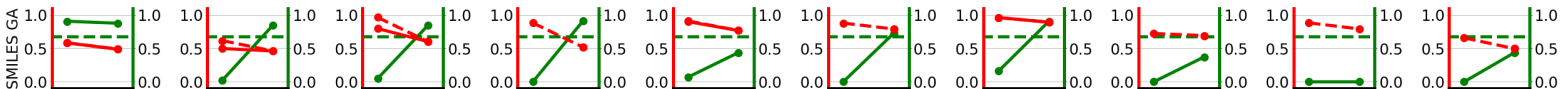

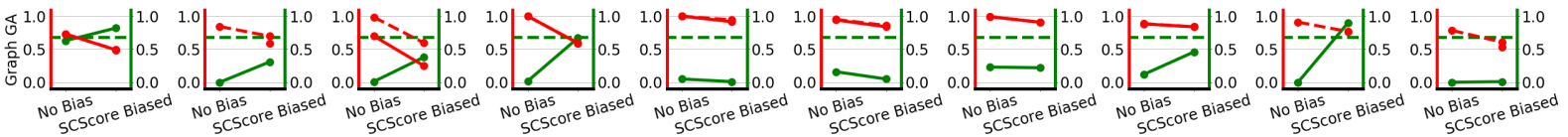

C Z Zaleplon MPO Sitagliptin MPO Valsartan SMARTS Scaffold Hop Fexofenadine MPO Osimertinib MPO Deco Hop Amlodipine MPO Ranolazine MPO Perindopril MPO

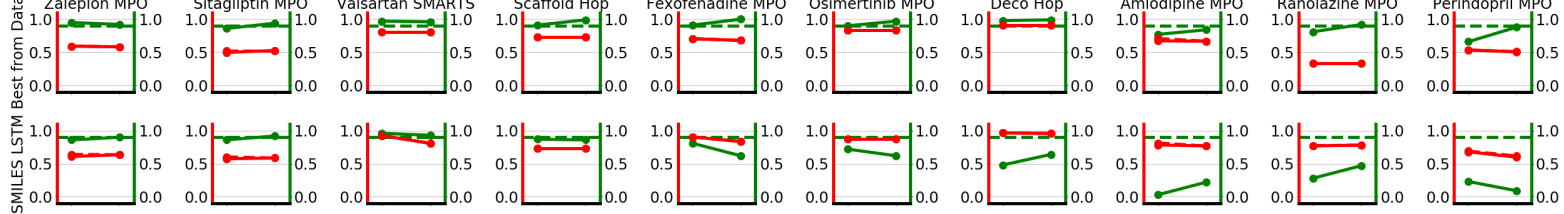

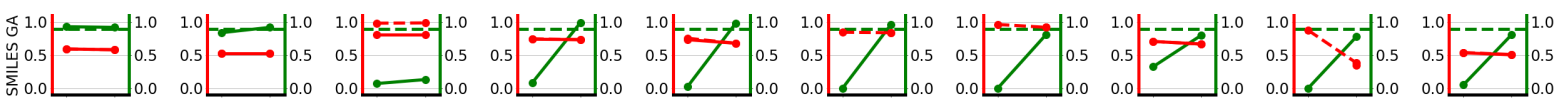

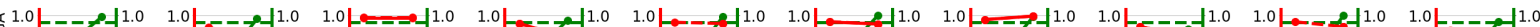
No Bias
SA_Score Biased

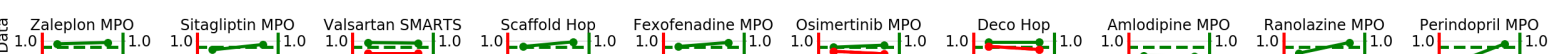

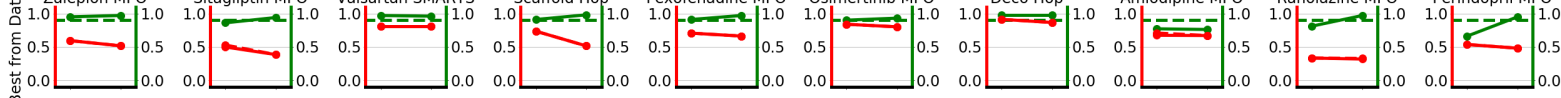

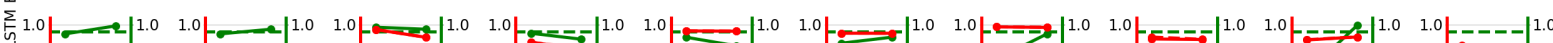

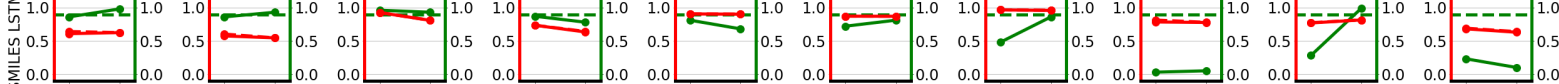

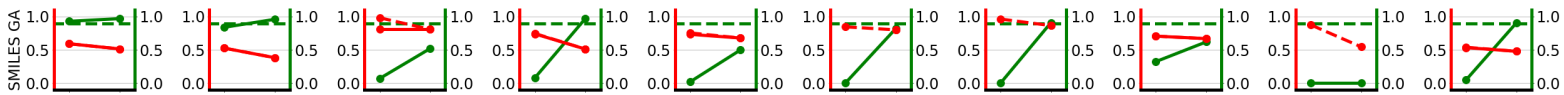

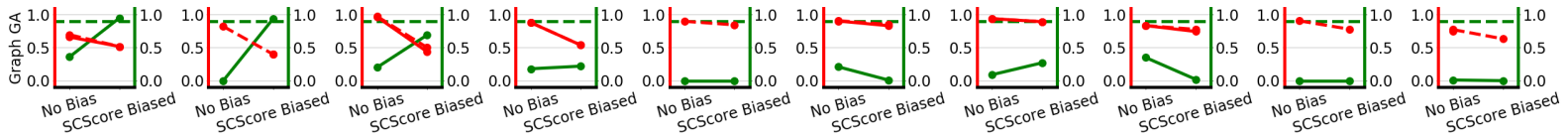
6

Figure S5: Change of synthesizability and objective function with heuristic biasing. (a) Biasing with SA_Score after training on ChEMBL; (b) biasing with SCScore after training on ChEMBL; (c) biasing with SA_Score after training on MOSES; (d) biasing with SCScore after training on MOSES. Within each panel, each row represents one generative method; each column represents one objective function. In each plot, the green solid line represents the change of fraction of synthesizable compounds in the top-100, with the green dashed line as a reference for the synthesizability of the training set (ChEMBL or MOSES). Red solid lines represent the change in the objective function value of the top synthesizable molecule, while the dashed red line represents the change in objective function value of the top molecule, regardless of its synthesizability. Plots without a solid red line indicates that no synthesizable structure was obtained in the top 100 molecules. All plots have a dashed red line, though it might be occluded by the solid line. 


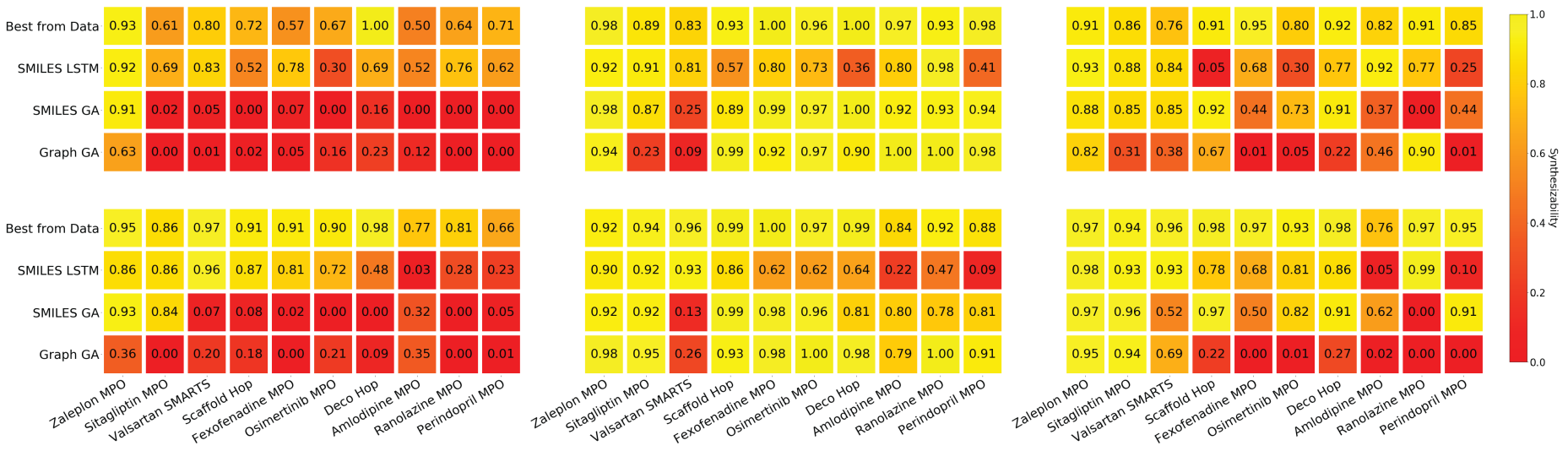

Figure S6: The synthesizable fraction of top-100 candidates proposed during goal-directed optimization for "hard" optimization tasks. (left) Without biasing; (middle) with heuristic biasing by SA_Score; (right) with heuristic biasing by SCScore. (top) using ChEMBL for initial training; (botom) using MOSES for initial training. For many tasks, ASKCOS is unable to identify routes to a large fraction of generated molecules, particularly when using the Graph GA or SMILES GA methods.

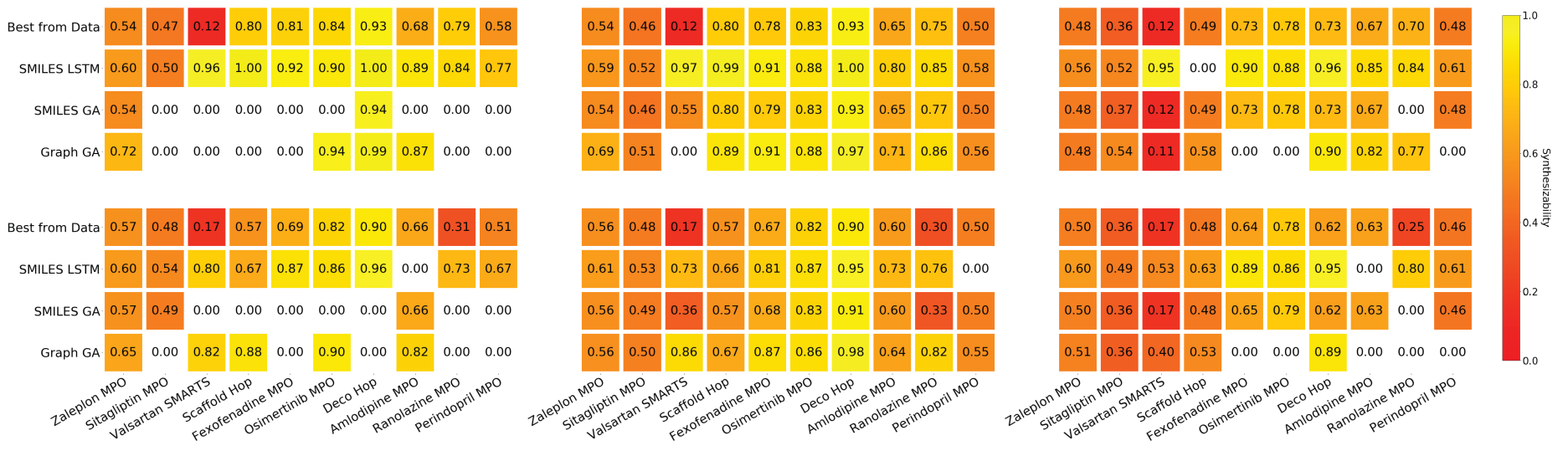

Figure S7: The average objective function value of the top-10 synthesizable candidates, identified through post hoc filtering of the top-100 candidates proposed during goal-directed optimization for "hard" optimization tasks. (left) Without biasing; (middle) with heuristic biasing by SA_Score; (right) with heuristic biasing by SCScore. (top) using ChEMBL for initial training; (botom) using MOSES for initial training. White squares with a value of 0.00 indicates that fewer than 10 of the top 100 molecules were identified as synthesizable by ASKCOS. The top row showing the Best from Dataset represents a virtual screening approach. 


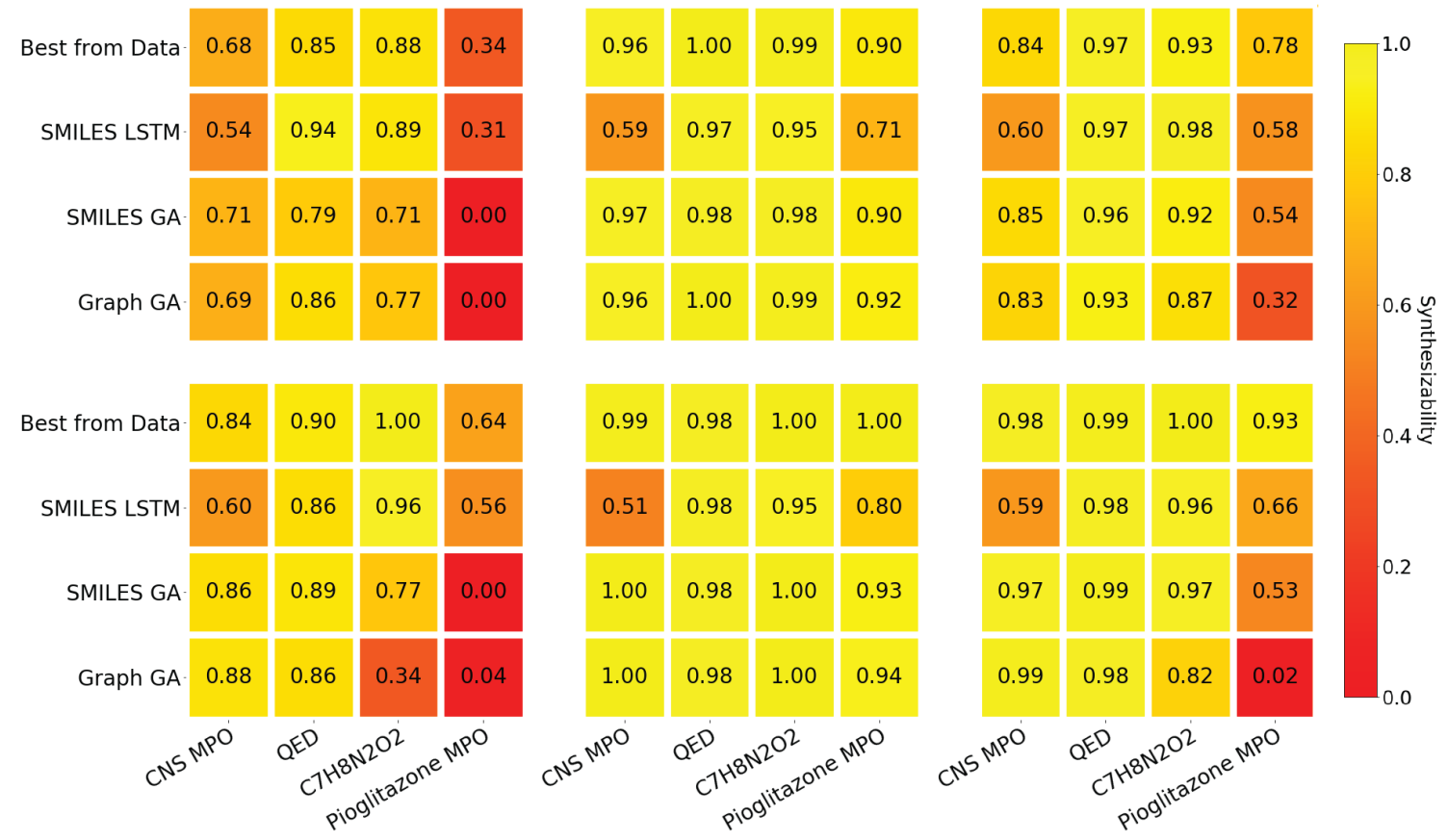

Figure S8: The synthesizable fraction of top-100 candidates proposed during goal-directed optimization for "trivial" optimization tasks. (left) Without biasing; (middle) with heuristic biasing by SA_Score; (right) with heuristic biasing by SCScore. (top) using ChEMBL for initial training; (botom) using MOSES for initial training. Synthesizability is primarily an issue for the Pioglitazone MPO task. 


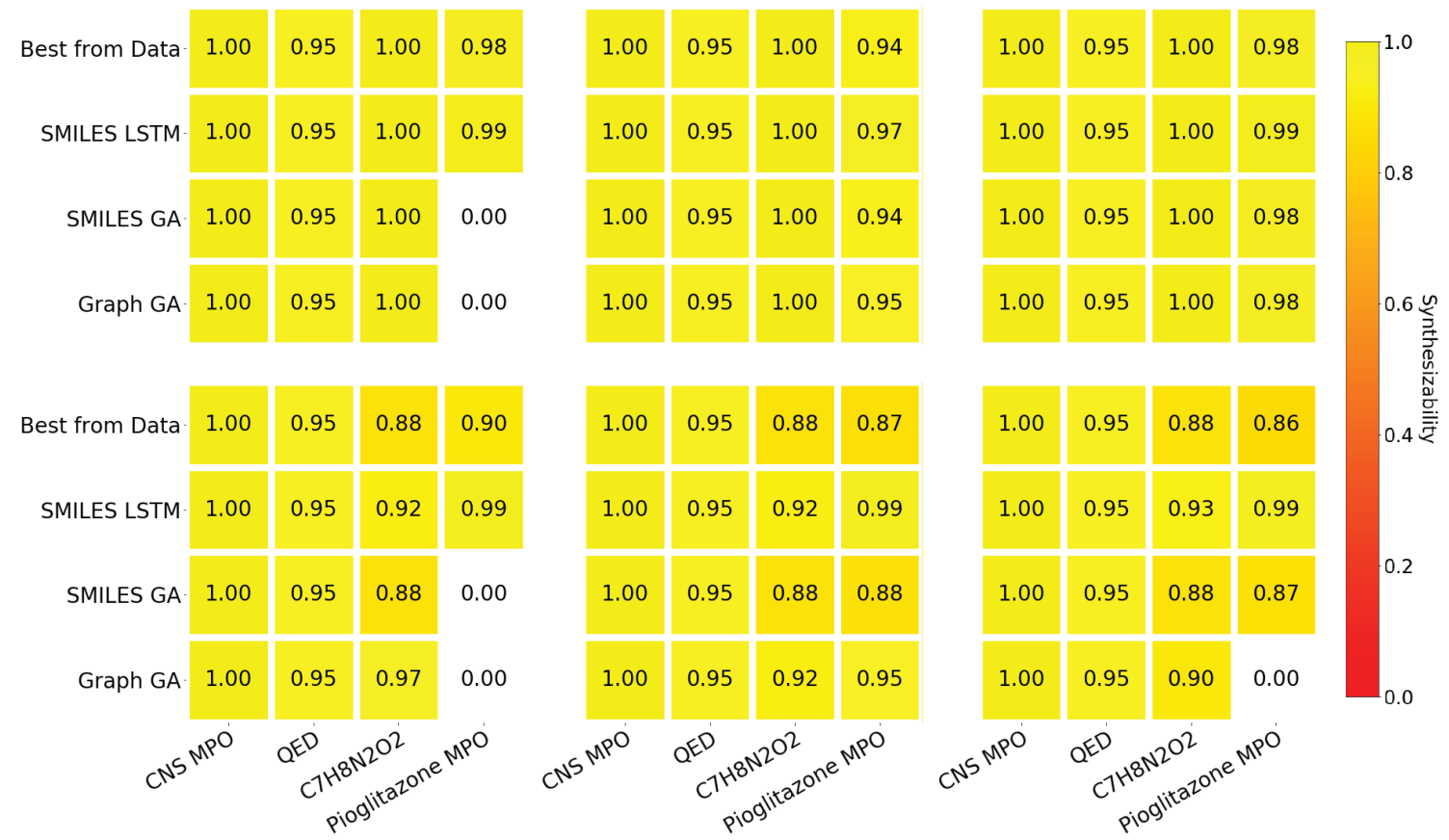

Figure S9: The average objective function value of the top-10 synthesizable candidates, identified through post hoc filtering of the top-100 candidates proposed during goal-directed optimization for "trivial" optimization tasks. (left) Without biasing; (middle) with heuristic biasing by SA_Score; (right) with heuristic biasing by SCScore. (top) using ChEMBL for initial training; (botom) using MOSES for initial training. White squares with a value of 0.00 indicates that fewer than 10 of the top 100 molecules were identified as synthesizable by ASKCOS. The top row showing the Best from Dataset represents a virtual screening approach. 
Successful cases of heuristic biasing in goal-directed generation 


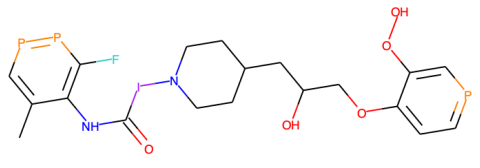

Ranolazine MPO smiles_ga: Top but unsynthesizable: 0.885

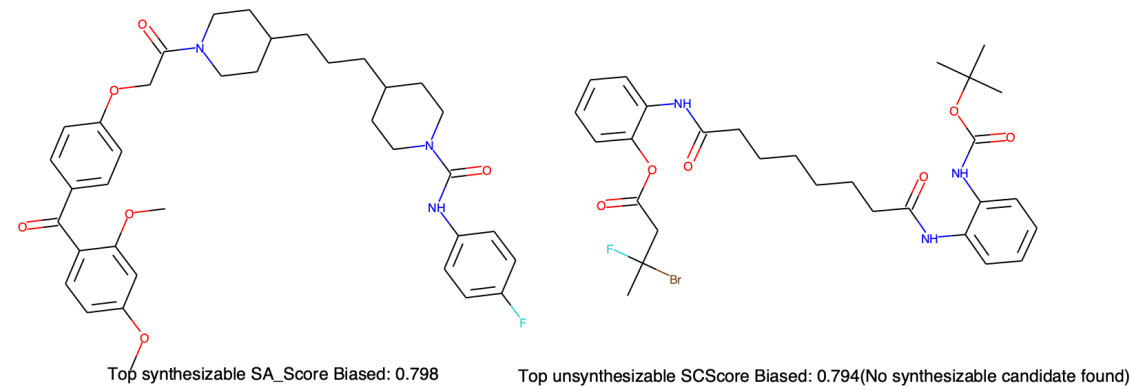

Top unsynthesizable SCScore Biased: 0.794(No synthesizable candidate found)

(a) SMILES GA / Ranolazine MPO / ChEMBL

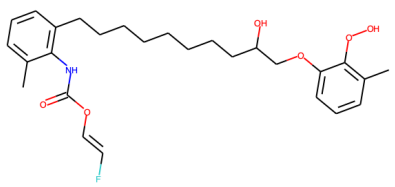

Ranolazine MPO graph_ga: Top but unsynthesizable: 0.910

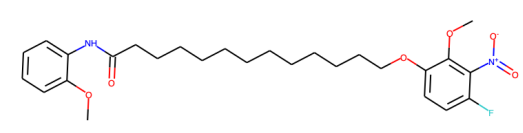

Top synthesizable SA_Score Biased: 0.867

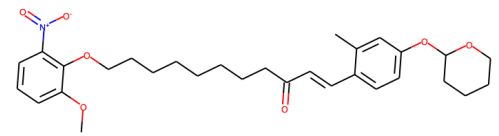

Top synthesizable SCScore Biased: 0.767

(b) Graph GA / Ranolazine MPO / ChEMBL

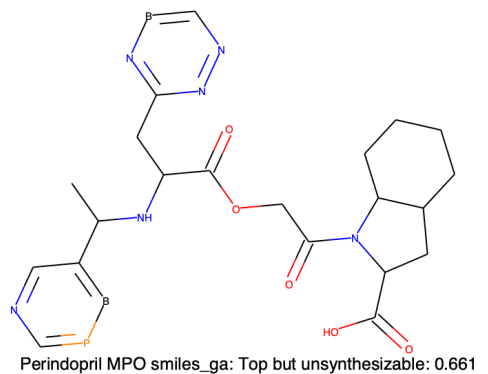

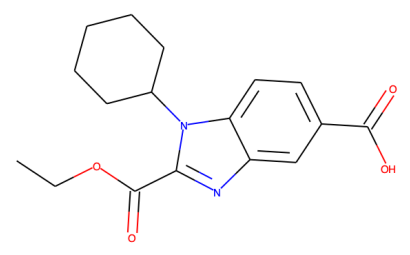

Top synthesizable SA_Score Biased: 0.509

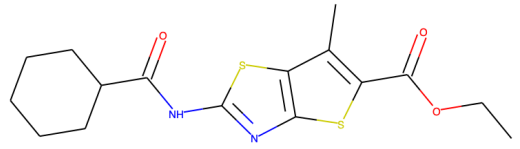

Top synthesizable SCScore Biased: 0.497

(c) SMILES GA / Perindopril MPO / ChEMBL

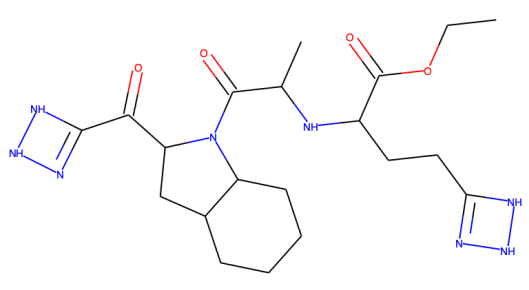

Perindopril MPO graph_ga: Top but unsynthesizable: 0.784

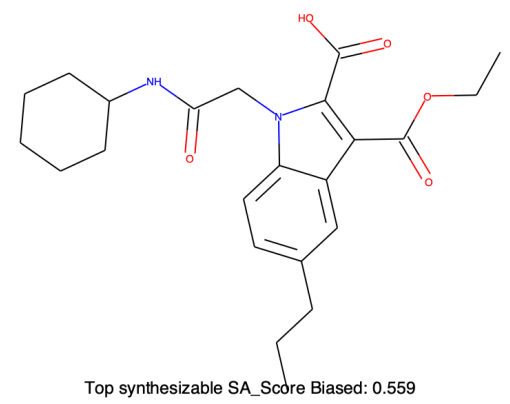

Top synthesizable SA_Score Biased: 0.559

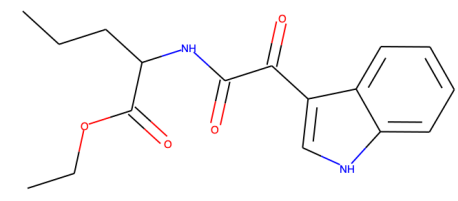

Top synthesizable SCScore Biased: 0.606

(d) Graph GA / Perindopril MPO / ChEMBL

11

Figure S10: Molecules proposed during goal-directed optimization where there are no synthesizable structures proposed in the top 100 candidates in the absence of heuristic biasing. Each row represents a particular method, objective function, and initial training set. From left to right, we draw the best (unsynthesizable) molecule, the best synthesizable molecule after biasing with the SA_Score, and the best synthesizable molecule after biasing with the SCScore. Many of the structures proposed by the SMILES GA and Graph GA methods are nonsensical and clearly unsynthesizable, despite achieving a high objective function value. 


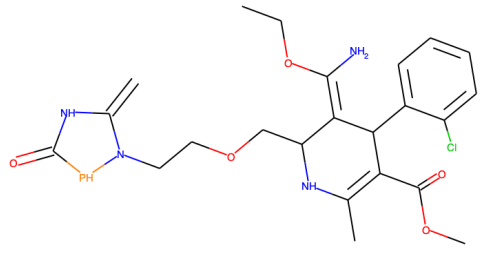

Amlodipine MPO smiles_ga: Top but unsynthesizable: 0.725

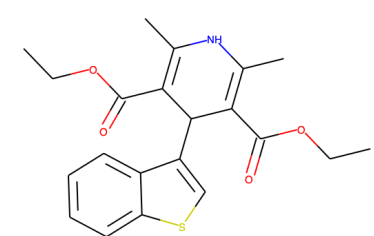

Top synthesizable SA_Score Biased: 0.689

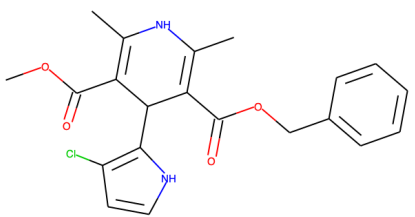

Top synthesizable SCScore Biased: 0.694

(a) SMILES GA / Amlodipine MPO / ChEMBL

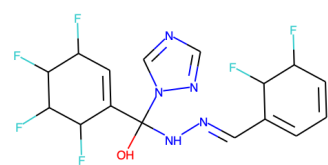

Sitagliptin MPO graph_ga: Top but unsynthesizable: 0.843

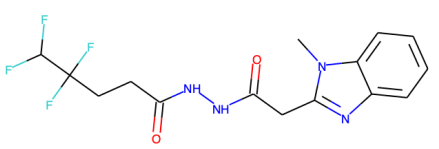

Top synthesizable SA Score Biased: 0.602

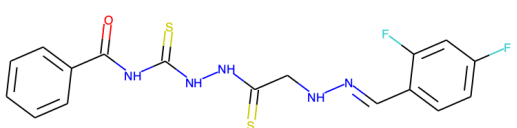

Top synthesizable SCScore Biased: 0.697

(b) Graph GA / Sitagliptin MPO / ChEMBL

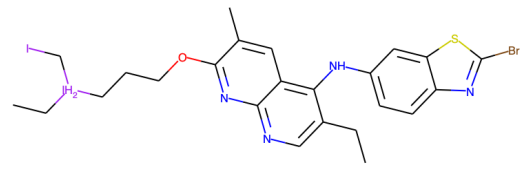

Scaffold Hop smiles_ga: Top but unsynthesizable: 0.885

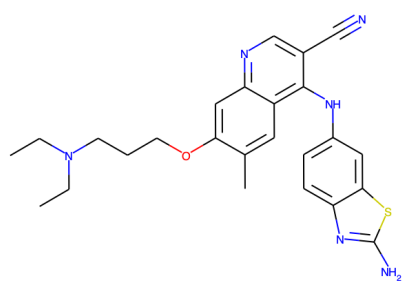

Top synthesizable SA_Score Biased: 0.827

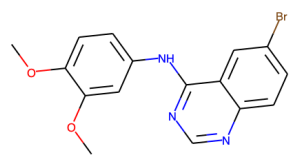

Top synthesizable SCScore Biased: 0.518

(c) SMILES GA / Scaffold Hop / ChEMBL

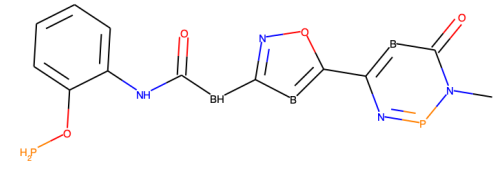

Osimertinib MPO smiles_ga: Top but unsynthesizable: 0.846

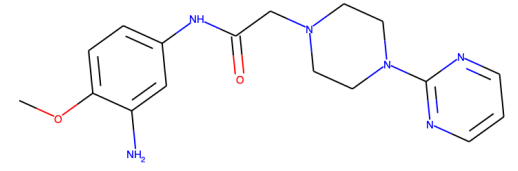

Top synthesizable SA_Score Biased: 0.838

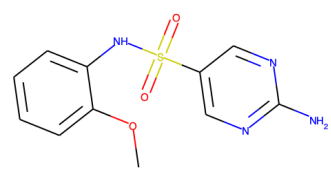

Top synthesizable SCScore Biased: 0.801

(d) SMILES GA / Osimertinib MPO / MOSES 12

Figure S11: Molecules proposed during goal-directed optimization where there are no synthesizable structures proposed in the top 100 candidates in the absence of heuristic biasing. Each row represents a particular method, objective function, and initial training set. From left to right, we draw the best (unsynthesizable) molecule, the best synthesizable molecule after biasing with the SA_Score, and the best synthesizable molecule after biasing with the SCScore. Many of the structures proposed by the SMILES GA and Graph GA methods are nonsensical and clearly unsynthesizable, despite achieving a high objective function value. 


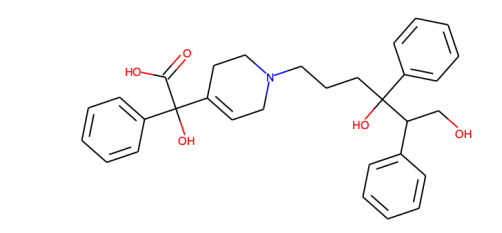

Fexofenadine MPO graph_ga: Top but unsynthesizable: 0.898

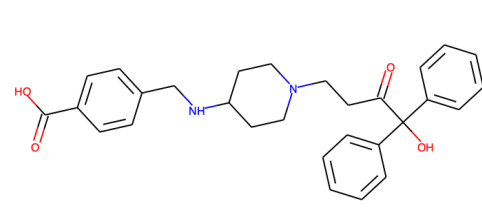

Top synthesizable SA_Score Biased: 0.878

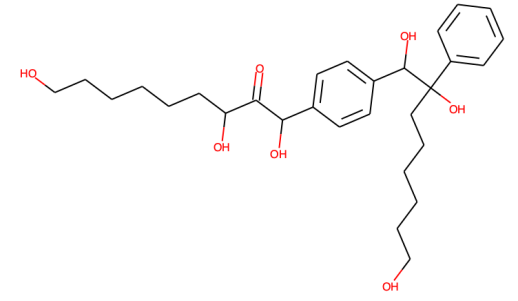

Top unsynthesizable SCScore Biased: 0.844(No synthesizable candidate found)

(a) Graph GA / Fexofenadine MPO / MOSES

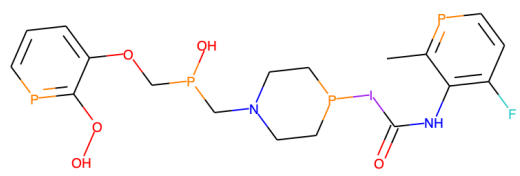

Ranolazine MPO smiles_ga: Top but unsynthesizable: 0.875

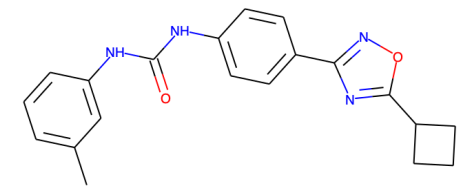

Top synthesizable SA_Score Biased: 0.381

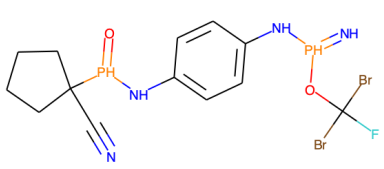

Top unsynthesizable SCScore Biased: 0.544(No synthesizable candidate found)

(b) SMILES GA / Ranolazine MPO / MOSES

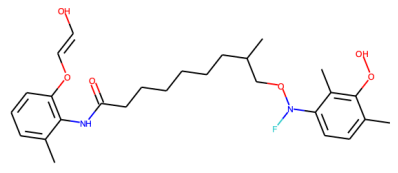

Ranolazine MPO graph_ga: Top but unsynthesizable: 0.905

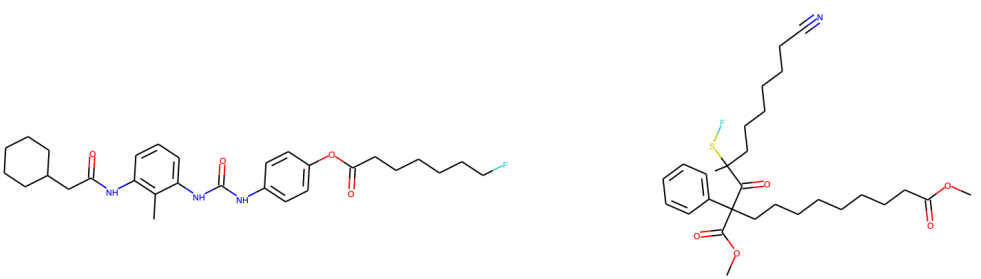

Top synthesizable SA_Score Biased: 0.829
Top unsynthesizable SCScore Biased: 0.778 (No synthesizable candidate found)

(c) Graph GA / Ranolazine MPO / MOSES

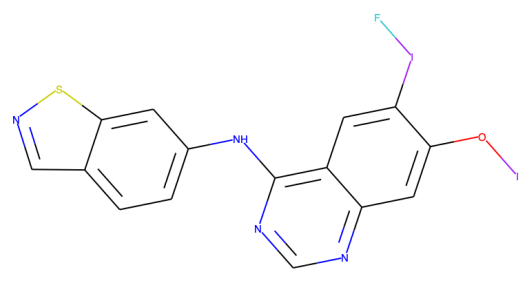

Deco Hop smiles_ga: Top but unsynthesizable: 0.963

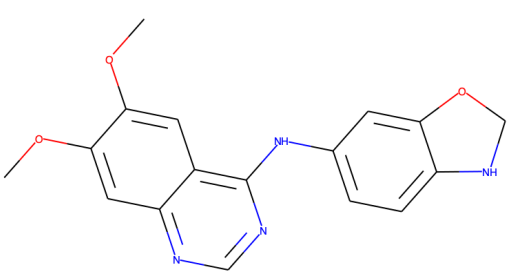

Top synthesizable SA_Score Biased: 0.920

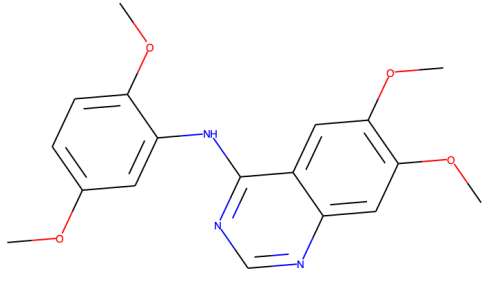

Top synthesizable SCScore Biased: 0.865

(d) SMILES GA / Decoration Hop / MOSES

13

Figure S12: Molecules proposed during goal-directed optimization where there are no synthesizable structures proposed in the top 100 candidates in the absence of heuristic biasing. Each row represents a particular method, objective function, and initial training set. From left to right, we draw the best (unsynthesizable) molecule, the best synthesizable molecule after biasing with the SA_Score, and the best synthesizable molecule after biasing with the SCScore. 


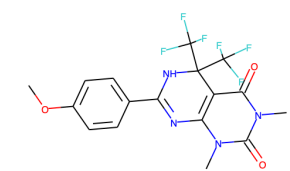

Sitagliptin MPO smiles_Istm: Top but unsynthesizable: 0.598

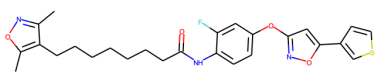

Ranolazine MPO smiles_ststm: Top but unsynthesizable: 0.772

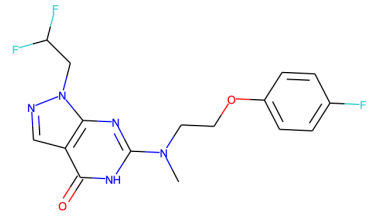

Sitagliptin MPO smiles_Istm: Top but unsynthesizable: 0.601

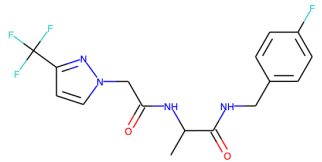

Top synthesizable: 0.516

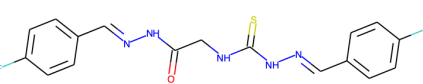

Top synthesizable SA_Score Biased: 0.580

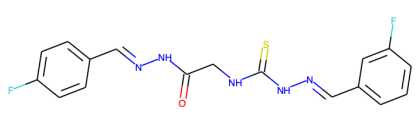

Top synthesizable SCScore Biased: 0.567

(a) SMILES LSTM / Sitagliptin MPO / ChEMBL

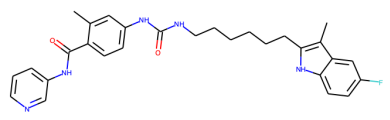

Top synthesizable: 0.768

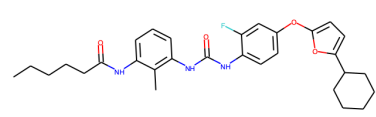

Top synthesizable SA_Score Biased: 0.781

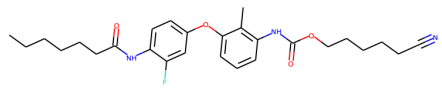

Top synthesizable SCScore Biased: 0.814

(b) SMILES LSTM / Ranolazine MPO / MOSES

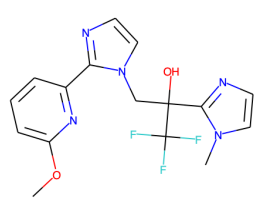

Top synthesizable: 0.573

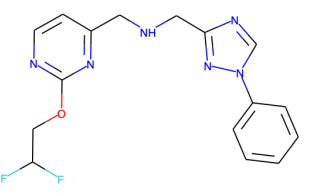

Top synthesizable SA_Score Biased: 0.585

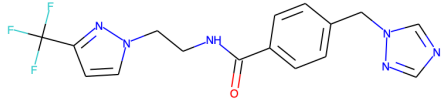

Top synthesizable SCScore Biased: 0.545

(c) SMILES LSTM / Sitagliptin MPO / MOSES

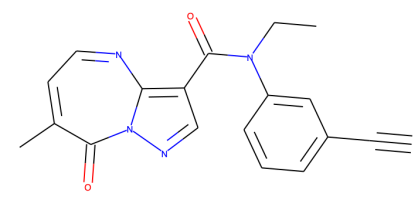

Zaleplon MPO smiles_Istm: Top but unsynthesizable: 0.639<smiles>C#Cc1cccc(C(CC)C(=C)C2=Cc3ccccc3C(C)C2=C)c1</smiles>

Top synthesizable: 0.608

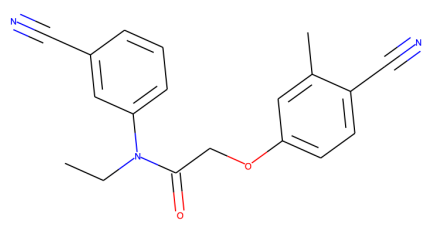

Top synthesizable SA_Score Biased: 0.635

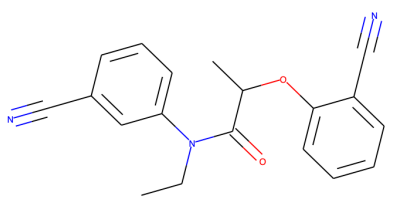

Top synthesizable SCScore Biased: 0.524

(d) SMILES LSTM / Zaleplon MPO / MOSES

Figure S13: Molecules proposed during goal-directed optimization where the main objective function value of the top-1 synthesizable structure is improved by heuristic biasing. Each row represents a particular method, objective function, and initial training set. From left to right, we draw the best (unsynthesizable) molecule, the best synthesizable molecule, the best synthesizable molecule after biasing with the SA_Score, and the best synthesizable molecule after biasing with the SCScore. All cases shown here use the SMILES LSTM method. 


\section{Agreement between ASKCOS results and synthesizability heuristics}

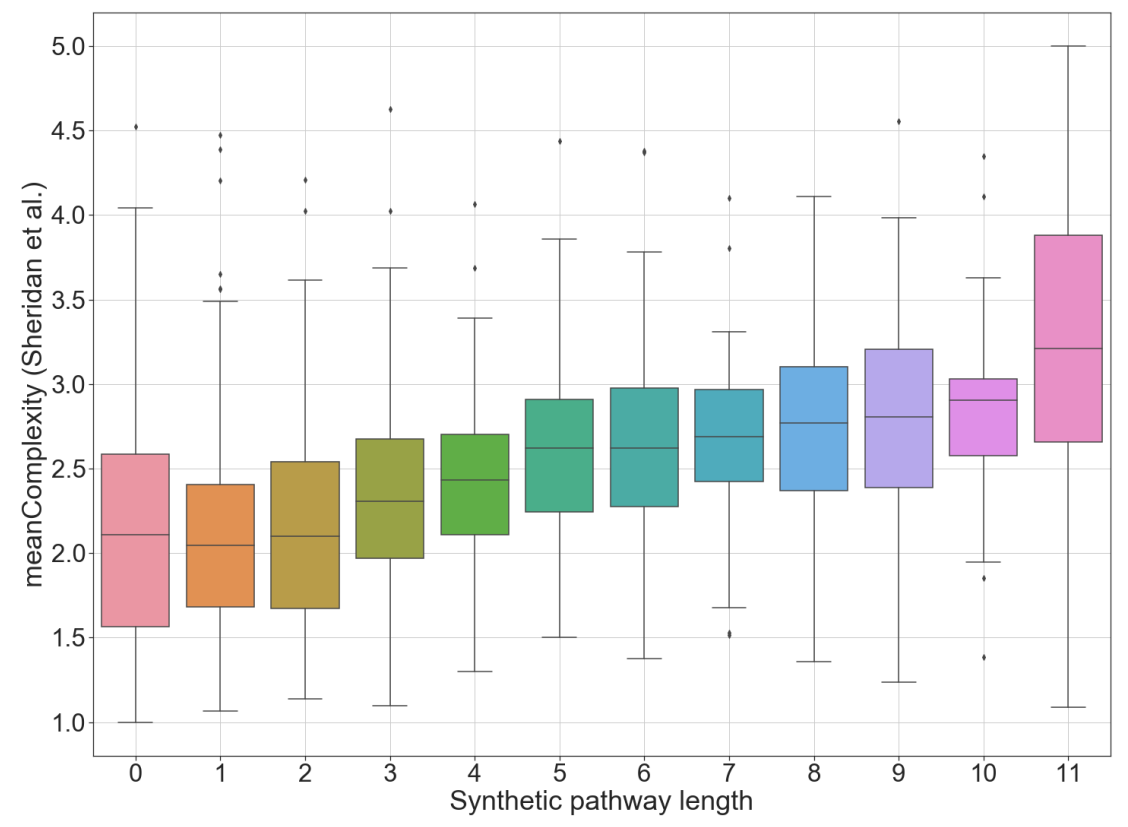

Figure S14: Correlation between the length of the first synthetic pathway found by ASKCOS and expert scores assigned by chemists in Sheridan et al. $17 \mathrm{~A}$ length of 0 indicates that the molecule can be found in our database of readily-purchasable compounds; a length of 11 indicates that no pathway was found with the fixed expansion settings (see Methods). 


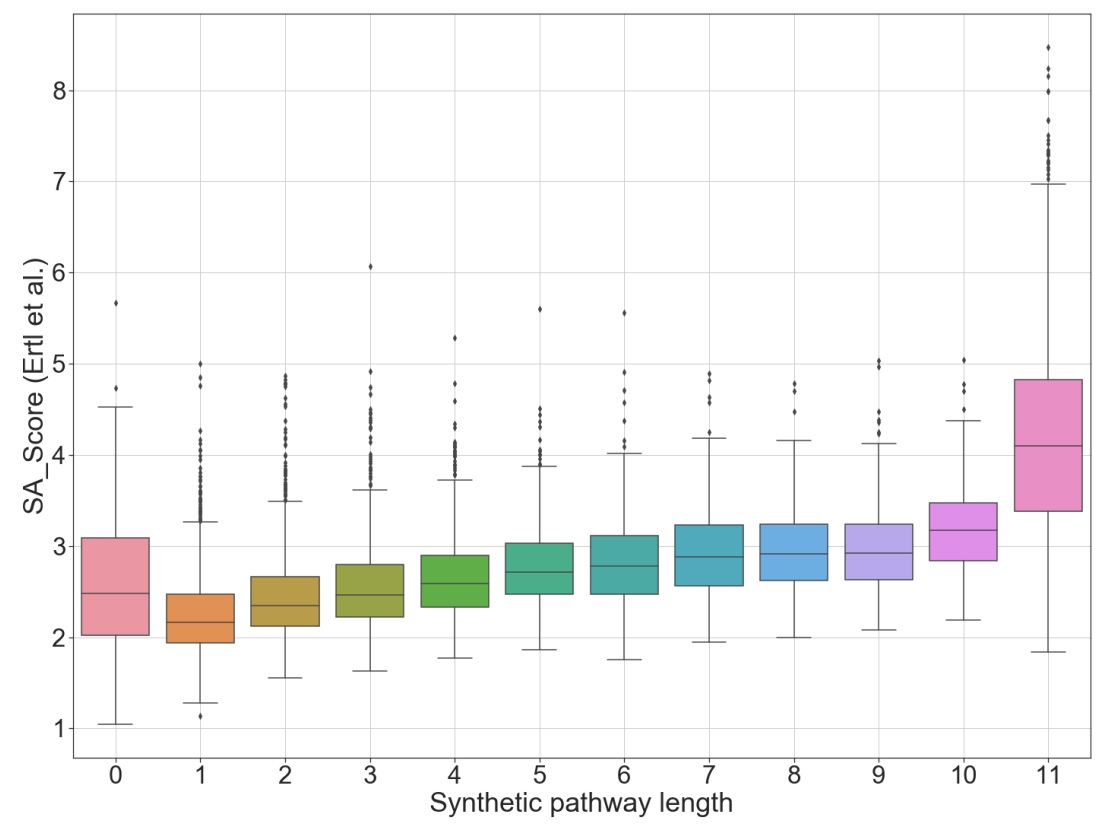

Figure S15: Correlation between the length of the first synthetic pathway found by ASKCOS using all compound datasets and the SA_Score ${ }^{31}$ heuristic. A length of 0 indicates that the molecule can be found in our database of readily-purchasable compounds; a length of 11 indicates that no pathway was found with the fixed expansion settings (see Methods). 


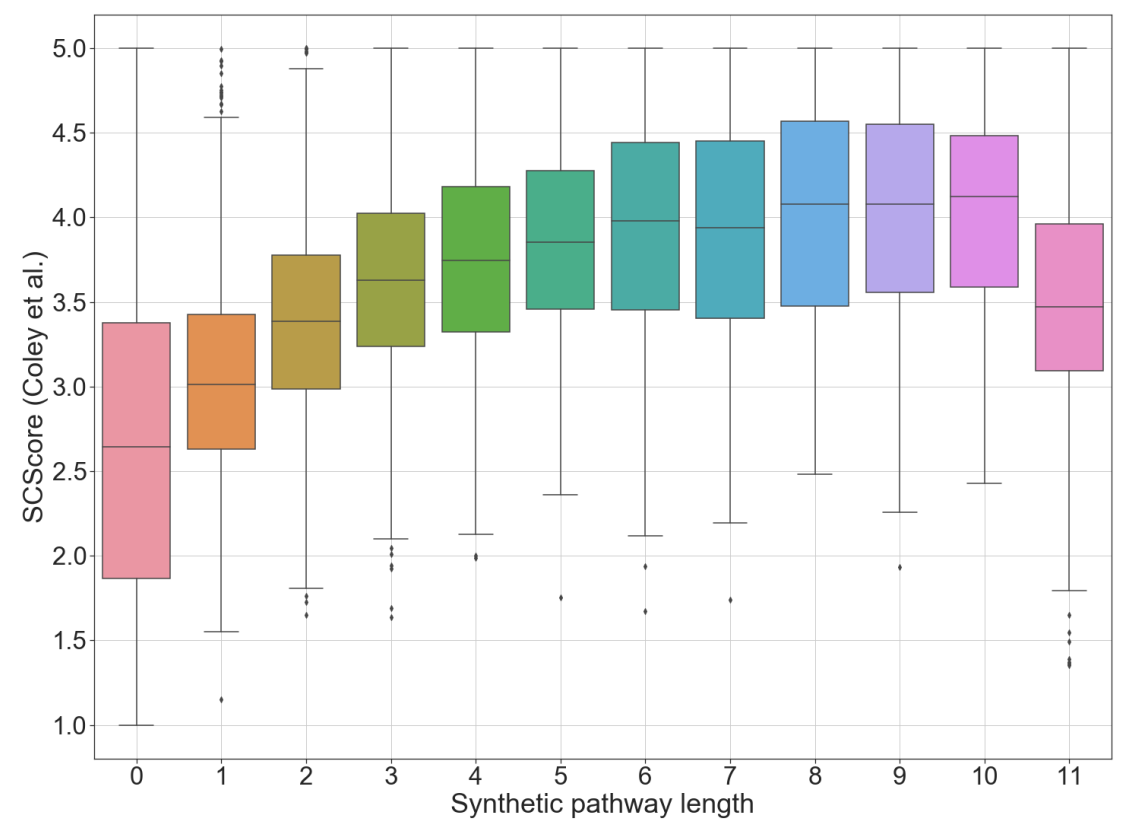

Figure S16: Correlation between the length of the first synthetic pathway found by ASKCOS using all compound datasets and the SCScore ${ }^{20}$ heuristic A length of 0 indicates that the molecule can be found in our database of readily-purchasable compounds; a length of 11 indicates that no pathway was found with the fixed expansion settings (see Methods). 


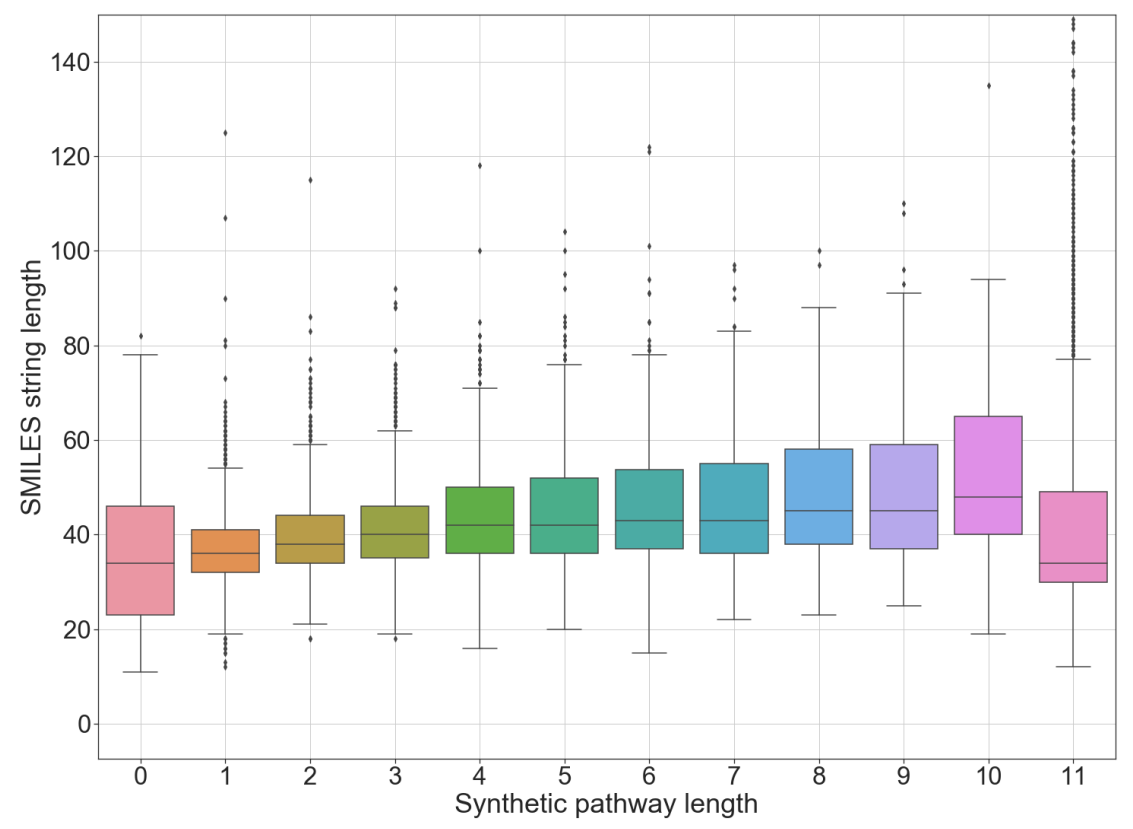

Figure S17: Correlation between the length of the first synthetic pathway found by ASKCOS using all compound datasets and the SMILES length heuristic A length of 0 indicates that the molecule can be found in our database of readily-purchasable compounds; a length of 11 indicates that no pathway was found with the fixed expansion settings (see Methods). 


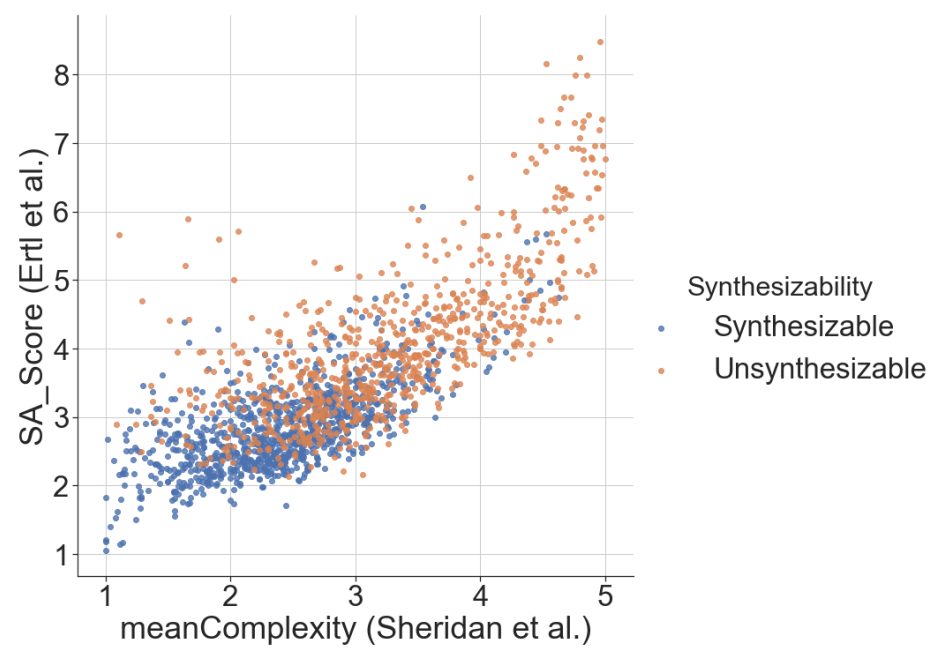

Figure S18: Correlation between Sheridan et al. 's meanComplexity 17 and the SA_Score; $; 1$ each compound is colored by its synthesizability according to ASKCOS.

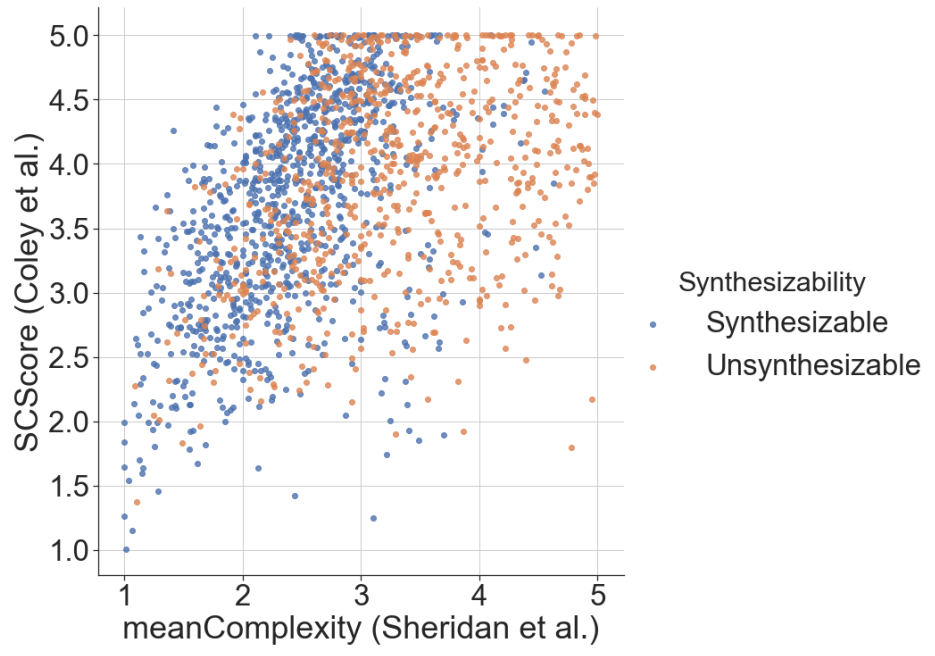

Figure S19: Correlation between Sheridan et al.'s meanComplexity 17 and the SCScore; 20 each compound is colored by its synthesizability according to ASKCOS. 


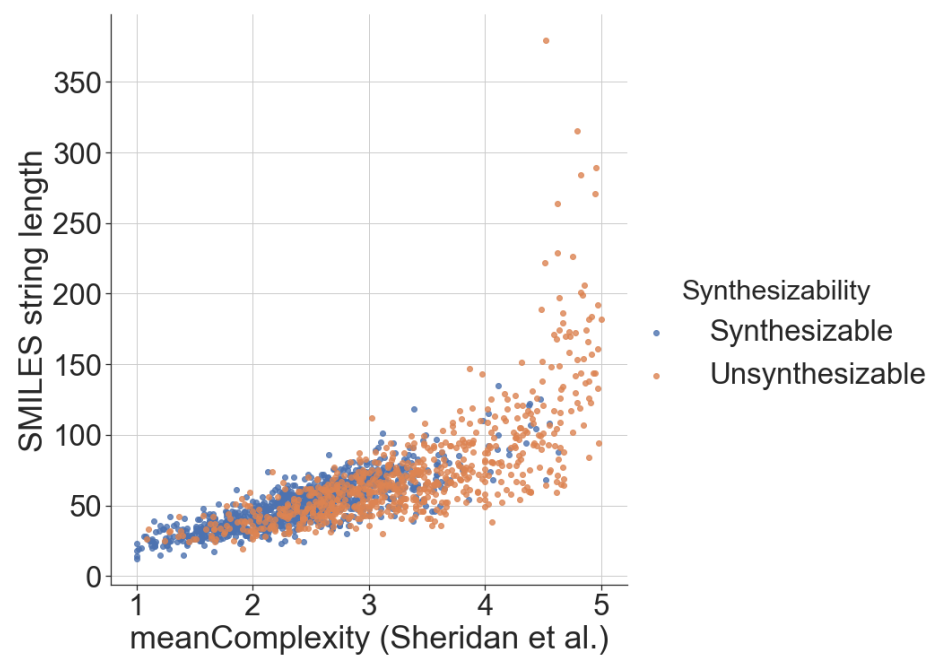

Figure S20: Correlation between Sheridan et al.'s meanComplexity 17 and the SMILES string length; each compound is colored by its synthesizability according to ASKCOS. 